\title{
Blow Up of the Solutions of Nonlinear Wave Equation in Reissner-Nordström Metric
}

\author{
Svetlin Georgiev
}

Communicated by Y. Charles Li, received January 23, 2006.

Abstract. In this paper we study the properties of the solutions to the Cauchy problem

$$
\begin{gathered}
\left(u_{t t}-\Delta u\right)_{g_{s}}=f(u)+g(|x|), \quad t \in[0,1], x \in \mathcal{R}^{3}, \\
u(1, x)=u_{0} \in \dot{B}_{p, q}^{\gamma}\left(\mathcal{R}^{3}\right), \quad u_{t}(1, x)=u_{1} \in \dot{B}_{p, q}^{\gamma-1}\left(\mathcal{R}^{3}\right),
\end{gathered}
$$

where $g_{s}$ is the Reissner-Nordström metric (see [2]); $p>1, q \geq 1, \gamma \in(0,1)$ are fixed constants, $f \in \mathcal{C}^{1}\left(\mathcal{R}^{1}\right), f(0)=0, a|u| \leq f^{\prime}(u) \leq b|u|, g \in \mathcal{C}\left(\mathcal{R}^{+}\right)$, $g(|x|) \geq 0, g(|x|)=0$ for $|x| \geq r_{1}, a$ and $b$ are positive constants, $r_{1}>0$ is suitable choosen.

When $g(r) \equiv 0$ we prove that the Cauchy problem (1), (2) has nontrivial solution $u \in \mathcal{C}\left((0,1] \dot{B}_{p, q}^{\gamma}\left(\mathcal{R}^{+}\right)\right)$in the form

$$
u(t, r)=\left\{\begin{array}{l}
v(t) \omega(r) \quad \text { for } \quad r \leq r_{1}, \quad t \in[0,1] \\
0 \quad \text { for } \quad r \geq r_{1}, \quad t \in[0,1]
\end{array}\right.
$$

where $r=|x|$, for which $\lim _{t \longrightarrow 0}\|u\|_{\dot{B}_{p, q}^{\gamma}\left(\mathcal{R}^{+}\right)}=\infty$.

When $g(r) \neq 0$ we prove that the Cauchy problem (1), (2) has nontrivial solution $u \in \mathcal{C}\left((0,1] \dot{B}_{p, q}^{\gamma}\left(\mathcal{R}^{+}\right)\right)$in the form

$$
u(t, r)=\left\{\begin{array}{l}
v(t) \omega(r) \quad \text { for } \quad r \leq r_{1}, \quad t \in[0,1] \\
0 \quad \text { for } \quad r \geq r_{1}, \quad t \in[0,1]
\end{array}\right.
$$

for which $\lim _{t \longrightarrow 0}\|u\|_{\dot{B}_{p, q}^{\gamma}\left(\mathcal{R}^{+}\right)}=\infty$.

\section{Introduction}

In this paper we study the properties of the solutions to the Cachy problem

$$
\begin{gathered}
\left(u_{t t}-\Delta u\right)_{g_{s}}=f(u)+g(|x|), \quad t \in[0,1], x \in \mathcal{R}^{3}, \\
u(1, x)=u_{0} \in \dot{B}_{p, q}^{\gamma}\left(\mathcal{R}^{3}\right), \quad u_{t}(1, x)=u_{1} \in \dot{B}_{p, q}^{\gamma-1}\left(\mathcal{R}^{3}\right),
\end{gathered}
$$

1991 Mathematics Subject Classification. 35L10, Secondary 35L50.

Key words and phrases. Blow up, nonlinear wave equation, Reissner-Nordström metric. 
where $g_{s}$ is the Reissner-Nordström metric (see [2])

$$
g_{s}=\frac{r^{2}-K r+Q^{2}}{r^{2}} d t^{2}-\frac{r^{2}}{r^{2}-K r+Q^{2}} d r^{2}-r^{2} d \phi^{2}-r^{2} \sin ^{2} \phi d \theta^{2},
$$

$K$ and $Q$ are positive constants, $p \in(1, \infty), q \geq 1$ and $\gamma \in(0,1)$ are fixed, $f \in \mathcal{C}^{1}\left(\mathcal{R}^{1}\right), f(0)=0, a|u| \leq f^{\prime}(u) \leq b|u|, g \in \mathcal{C}\left(\mathcal{R}^{+}\right), g(|x|) \geq 0, g(|x|)=0$ for $|x| \geq r_{1}, a$ and $b$ are positive constants, $r_{1}>0$ is suitable choosen.

The Cauchy problem (1), (2) we may rewrite in the form

$$
\begin{gathered}
\frac{r^{2}}{r^{2}-K r+Q^{2}} u_{t t}-\frac{1}{r^{2}} \partial_{r}\left(\left(r^{2}-K r+Q^{2}\right) u_{r}\right)- \\
\frac{1}{r^{2} \sin \phi} \partial_{\phi}\left(\sin \phi u_{\phi}\right)-\frac{1}{r^{2} \sin ^{2} \phi} u_{\theta \theta}=f(u)+g(r), \\
u(1, r, \phi, \theta)=u_{0} \in \dot{B}_{p, q}^{\gamma}\left(\mathcal{R}^{+} \times[0,2 \pi] \times[0, \pi]\right), \\
u_{t}(1, r, \phi, \theta)=u_{1} \in \dot{B}_{p, q}^{\gamma-1}\left(\mathcal{R}^{+} \times[0,2 \pi] \times[0, \pi]\right) .
\end{gathered}
$$

When $g_{s}$ is the Minkowski metric; $u_{0}, u_{1} \in \mathcal{C}_{0}^{\infty}\left(\mathcal{R}^{3}\right)$ in [6] (see and [1], section $6.3)$ is proved that there exists $T>0$ and a unique local solution $u \in \mathcal{C}^{2}\left([0, T) \times \mathcal{R}^{3}\right)$ for the Cauchy problem

$$
\begin{gathered}
\left(u_{t t}-\Delta u\right)_{g_{s}}= \\
f(u), \quad f \in \mathcal{C}^{2}(\mathcal{R}), \quad t \in[0, T], x \in \mathcal{R}^{3}, \\
\left.u\right|_{t=0}=u_{0},\left.u_{t}\right|_{t=0}=u_{1},
\end{gathered}
$$

for which

$$
\sup _{t<T, x \in \mathcal{R}^{3}}|u(t, x)|=\infty .
$$

When $g_{s}$ is the Minkowski metric, $1 \leq p<5$ and initial data are in $\mathcal{C}_{0}^{\infty}\left(\mathcal{R}^{3}\right)$, in [6] (see and [1], section 6.3) is proved that the initial value problem

$$
\begin{gathered}
\left(u_{t t}-\Delta u\right)_{g_{s}}=u|u|^{p-1}, \quad t \in[0, T], x \in \mathcal{R}^{3}, \\
\left.u\right|_{t=0}=u_{0},\left.u_{t}\right|_{t=0}=u_{1},
\end{gathered}
$$

admits a global smooth solution.

When $g_{s}$ is the Minkowski metric and initial data are in $\mathcal{C}_{0}^{\infty}\left(\mathcal{R}^{3}\right)$, in [5] (see and [1], section 6.3) is proved that there exists a number $\epsilon_{0}>0$ such that for any data $\left(u_{0}, u_{1}\right) \in \mathcal{C}_{0}^{\infty}\left(\mathcal{R}^{3}\right)$ with $E(u(0))<\epsilon_{0}$, the initial value problem

$$
\begin{aligned}
\left(u_{t t}-\Delta u\right)_{g_{s}} & =u^{5}, \quad t \in[0, T], x \in \mathcal{R}^{3}, \\
\left.u\right|_{t=0} & =u_{0},\left.u_{t}\right|_{t=0}=u_{1},
\end{aligned}
$$

admits a global smooth solution.

When $g_{s}$ is the Reissner-Nordström metrics in [4] is proved that the Cauchy problem

$$
\begin{gathered}
\left(u_{t t}-\Delta u\right)_{g_{s}}+m^{2} u=f(u), \quad t \in[0,1], x \in \mathcal{R}^{3}, \\
u(1, x)=u_{0} \in \dot{B}_{p, p}^{\gamma}\left(\mathcal{R}^{3}\right), \quad u_{t}(1, x)=u_{1} \in \dot{B}_{p, p}^{\gamma-1}\left(\mathcal{R}^{3}\right),
\end{gathered}
$$

where $m \neq 0$ is constant and $f \in \mathcal{C}^{2}\left(\mathcal{R}^{2}\right), a|u| \leq\left|f^{(l)}(u)\right| \leq b|u|, l=0,1, a$ and $b$ are positive constants, has unique nontrivial solution $u(t, r) \in \mathcal{C}\left((0,1] \dot{B}_{p, p}^{\gamma}\left(\mathcal{R}^{+}\right)\right)$, $r=|x|, p>1$, for which

$$
\lim _{t \longrightarrow 0}\|u\|_{\dot{B}_{p, p}^{\gamma}\left(\mathcal{R}^{+}\right)}=\infty .
$$


When $g_{s}$ is the Minkowski metric in [7] is proved that the Cauchy problem

$$
\begin{gathered}
\left(u_{t t}-\Delta u\right)_{g_{s}}=f(u), \quad t \in[0,1], x \in \mathcal{R}^{3}, \\
u(1, x)=u_{0}, \quad u_{t}(1, x)=u_{1},
\end{gathered}
$$

has global solution. Here $f \in \mathcal{C}^{2}(\mathcal{R}), f(0)=f^{\prime}(0)=f^{\prime \prime}(0)=0$,

$$
\left|f^{\prime \prime}(u)-f^{\prime \prime}(v)\right| \leq B|u-v|^{q_{1}}
$$

for $|u| \leq 1,|v| \leq 1, B>0, \sqrt{2}-1<q_{1} \leq 1, u_{0} \in \mathcal{C}_{\circ}^{5}\left(\mathcal{R}^{3}\right), u_{1} \in \mathcal{C}_{\circ}^{4}\left(\mathcal{R}^{3}\right)$, $u_{0}(x)=u_{1}(x)=0$ for $\left|x-x_{0}\right|>\rho, x_{0}$ and $\rho$ are suitable choosen.

Our main results are

Theorem 1. Let $p>1, q \geq 1, \gamma \in(0,1)$ and $K, Q$ are positive constants for which

$$
\left\{\begin{array}{l}
K^{2}>4 Q^{2}, \quad \frac{1}{1-K+Q^{2}} \geq 1, \\
1-K+Q^{2}>0 \text { is small enough such that } \\
\frac{K-\sqrt{K^{2}-4 Q^{2}}}{2}-2 \sqrt{1-K+Q^{2}}>0 .
\end{array}\right.
$$

Let also $g \equiv 0, f \in \mathcal{C}^{1}\left(\mathcal{R}^{1}\right), f(0)=0, a|u| \leq f^{\prime}(u) \leq b|u|$, a and $b$ are positive constants. Then the homogeneous Cauchy problem (1), (2) has nontrivial solution $u(t, r)=v(t) \omega(r) \in \mathcal{C}\left((0,1] \dot{B}_{p, q}^{\gamma}\left(\mathcal{R}^{+}\right)\right)$for which

$$
\lim _{t \longrightarrow 0}\|u\|_{\dot{B}_{p, q}^{\gamma}\left(\mathcal{R}^{+}\right)}=\infty \text {. }
$$

Theorem 2. Let $p>1, q \geq 1, \gamma \in(0,1)$ and $K, Q$ are positive constants for which

$$
\left\{\begin{array}{l}
K^{2}>4 Q^{2}, \quad \frac{1}{1-K+Q^{2}} \geq 1 \\
1-K+Q^{2}>0 \text { is small enough such that } \\
\frac{K-\sqrt{K^{2}-4 Q^{2}}}{2}-2 \sqrt{1-K+Q^{2}}>0 .
\end{array}\right.
$$

Let also $g \neq 0, g \in \mathcal{C}\left(\mathcal{R}^{+}\right), g(r) \geq 0$ for $r \geq 0, g(r)=0$ for $r \geq r_{1}, f \in \mathcal{C}^{1}\left(\mathcal{R}^{1}\right)$, $f(0)=0, a|u| \leq f^{\prime}(u) \leq b|u|, a$ and $b$ are positive constants. Then the nonhomogeneous Cauchy problem (1), (2) has nontrivial solution $u(t, r)=v(t) \omega(r) \in$ $\mathcal{C}\left((0,1] \dot{B}_{p, q}^{\gamma}\left(\mathcal{R}^{+}\right)\right)$for which

$$
\lim _{t \longrightarrow 0}\|u\|_{\dot{B}_{p, q}^{\gamma}\left(\mathcal{R}^{+}\right)}=\infty .
$$

The paper is organized as follows. In section 2 we will prove some preliminary results. In section 3 we prove theorem 1 . In section 4 we prove theorem 2 . In appendix we prove some results which are used in the proof of theorem 1 and theorem 2 .

\section{Preliminary results}

For fixed $q \geq 1$ and $\gamma \in(0,1)$ we put

$$
C=\left(\frac{q \gamma 2^{q \gamma}}{2^{q \gamma}-1}\right)^{\frac{1}{q}}
$$

For fixed $p>1, q \geq 1, \gamma \in(0,1)$ and $g \in \mathcal{C}\left(\mathcal{R}^{+}\right), g(r) \geq 0$ for $r \geq 0$ we suppose that the constants $A>0, Q>0, a>0, b>0, B>0, K>0,1<\beta<\alpha$ satisfy 
the conditions

$$
\begin{aligned}
& \text { i1) } \frac{1}{1-K+Q^{2}}\left(\frac{1}{1-K+Q^{2}} \frac{2 a}{A^{2}}+\frac{b}{A B}\right) \leq 1, \quad A>1, \quad \frac{A^{2}}{a}>1 ; \\
& \left\{\begin{array}{l}
\frac{1}{\alpha^{2}\left(1-\alpha K+\alpha^{2} Q^{2}\right)} \frac{a}{2 A^{4}}-\frac{b r_{1}^{2}}{A B} \geq 0, \\
\frac{a}{2 A^{6} \alpha^{2}\left(1-\alpha K+\alpha^{2} Q^{2}\right)}-\frac{2 b r_{1}^{2}}{A^{2} B^{2}} \geq 0,
\end{array}\right. \\
& \text { i2) }\left\{\begin{array}{l}
\frac{a}{2 A^{4}\left(1-\alpha K+\alpha^{2} Q^{2}\right)}\left(\frac{1}{\beta}-\frac{1}{\alpha}\right)-\frac{4 a r_{1}}{A^{3} B\left(1-K+Q^{2}\right)^{2}} \geq 0, \\
\left(\frac{1}{\beta}-\frac{1}{\alpha}\right)^{2} \quad 1
\end{array}\right. \\
& \left(\frac{1}{\beta}-\frac{1}{\alpha}\right)^{2} \frac{1}{\left(1-\alpha K+\alpha^{2} Q^{2}\right)^{2}} \frac{a}{4 A^{6}} \\
& -r_{1}^{2} \frac{2 b}{\left(1-K+Q^{2}\right) A^{2} B^{2}}-r_{1}^{2} \frac{1}{1-K+Q^{2}} \max _{r \in\left[0, r_{1}\right]} g(r) \geq \frac{1}{A^{2}}, \\
& \left(\frac{1}{\beta}-\frac{1}{\alpha}\right)^{2} \frac{1}{\left(1-\alpha K+\alpha^{2} Q^{2}\right)^{2}} \frac{a}{2 A^{4}}-\frac{2 a r_{1}^{4}}{A B\left(1-K+Q^{2}\right)}>0, \\
& \text { i3) } C\left(\frac{1}{\left(1-K+Q^{2}\right)^{2}} \frac{2 a}{A^{2}}+\frac{2 b}{A B\left(1-K+Q^{2}\right)}+\frac{1}{A^{2}}\right) \frac{2^{2-\gamma}}{(q(1-\gamma))^{\frac{1}{q}}}+ \\
& C \frac{2^{1-\gamma}}{A^{2}\left(1-K+Q^{2}\right)^{2}(q(1-\gamma))^{\frac{1}{q}}}<1,
\end{aligned}
$$

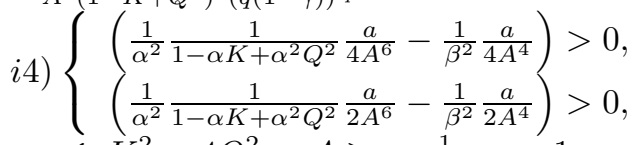

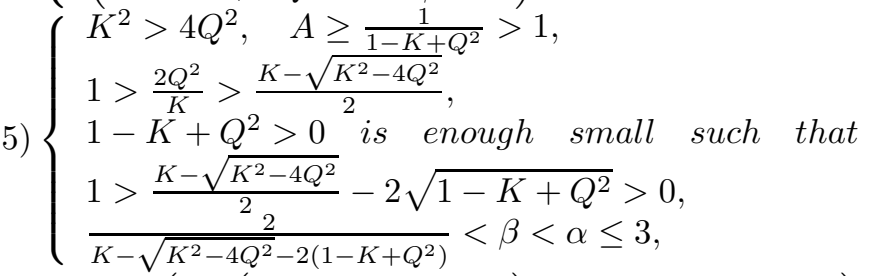

$$
\begin{aligned}
& \text { i6) } \frac{1}{1-K+Q^{2}}\left(\frac{2}{A B}\left(\frac{1}{1-K+Q^{2}} \frac{2 a}{A^{2}}+\frac{b}{A B}\right)+r_{1}^{2} \max _{s \in\left[0, r_{1}\right]} g(s)\right) \leq \frac{2}{A B} \text {; } \\
& \text { i7) } \max _{s \in\left[0, r_{1}\right]} g(s) \leq\left(\frac{1}{\beta}-\frac{1}{\alpha}\right)^{2} \frac{1}{A^{4}} \text {, }
\end{aligned}
$$

where

$$
r_{1}=\frac{K-\sqrt{K^{2}-4 Q^{2}}}{2}-\sqrt{1-K+Q^{2}} .
$$

Example. Let $0<\epsilon<<\frac{1}{3}$ is enough small,

$$
\begin{aligned}
& A=\frac{1}{\epsilon^{4}}, \quad B=\frac{1}{\epsilon}, \quad p=\frac{3}{2}, \quad q=\frac{3}{2}, \quad \gamma=\frac{1}{3}, \quad \alpha=3, \\
& \frac{1}{\beta}=\frac{K-\sqrt{K^{2}-4 Q^{2}}}{2}-2 \sqrt{1-K+Q^{2}}, \\
& g(r)=\left\{\begin{array}{l}
\epsilon^{11}\left(r-r_{1}\right)^{2} \quad \text { for } r \leq r_{1}, \\
0 \text { for } r \geq r_{1},
\end{array}\right. \\
& K=\frac{4}{3}+\frac{1}{6} \epsilon^{20}-\frac{3}{2} \epsilon^{2}, \\
& Q^{2}=\frac{1}{3}+\frac{1}{6} \epsilon^{20}-\frac{1}{2} \epsilon^{2} \text {, } \\
& a=\epsilon^{4}, \quad b=\epsilon^{3} \text {. }
\end{aligned}
$$

Then

$$
\begin{aligned}
& 1-\alpha K+\alpha^{2} Q^{2}=1-3 K+9 Q^{2}=\epsilon^{20} \\
& 1-K+Q^{2}=\epsilon^{2} \bullet
\end{aligned}
$$

When $g(r) \equiv 0$ we put

$$
u_{0}:=v(1) \omega(r)=
$$




$$
=\left\{\begin{array}{l}
\int_{r}^{r_{1}} \frac{1}{\tau^{2}-K \tau+Q^{2}} \int_{\tau}^{r_{1}}\left(\frac{s^{4}}{s^{2}-K s+Q^{2}} v^{\prime \prime}(1) \omega(s)-s^{2} f(v(1) \omega(s))\right) d s d \tau \\
\quad \text { for } r \leq r_{1}, \\
0 \quad \text { for } r \geq r_{1},
\end{array}\right.
$$

and $u_{1} \equiv 0$. Here $v(t)$ is fixed function which satisfies the conditions

$(H 1) \quad v(t) \in \mathcal{C}^{3}[0, \infty), \quad v(t)>0 \quad$ for $\quad \forall t \in[0,1]$;

$(H 2) \quad v^{\prime \prime}(t)>0 \quad$ for $\quad \forall t \in[0,1], \quad v^{\prime}(1)=v^{\prime \prime \prime}(1)=0, \quad v(1) \neq 0$;

(H3) $\left\{\min _{t \in[0,1]} \frac{v^{\prime \prime}(t)}{v(t)} \geq \frac{a}{2 A^{4}}, \quad \max _{t \in[0,1]} \frac{v^{\prime \prime}(t)}{v(t)} \leq \frac{2 a}{A^{2}}\right.$;

$\lim _{t \longrightarrow 0}\left[v^{\prime \prime}(t)-\frac{a}{2 A^{4}} v(t)\right]=+0, \quad v^{\prime \prime}(t)-\frac{a}{2 A^{4}} v(t) \geq 0 \quad$ for $\quad t \in[0,1]$.

In section 3 we will prove that the equation $\left(1^{\prime}\right)$ has unique nontrivial solution $\omega(r)$ for which $\omega(r) \in \mathcal{C}^{2}\left[0, r_{1}\right], \omega(r) \in \dot{B}_{p, q}^{\gamma}\left[0, r_{1}\right],|\omega(r)| \leq \frac{2}{A B}$ for $r \in\left[0, r_{1}\right], \omega(r) \geq \frac{1}{A^{2}}$ for $r \in\left[\frac{1}{\alpha}, \frac{1}{\beta}\right], \omega\left(r_{1}\right)=\omega^{\prime}\left(r_{1}\right)=\omega^{\prime \prime}\left(r_{1}\right)=0$.

When $g(r) \neq 0$ we put

$$
u_{0}=v(1) \omega(r)=
$$

$$
=\left\{\begin{array}{l}
\int_{r}^{r_{1}} \frac{1}{\tau^{2}-K \tau+Q^{2}} \int_{\tau}^{r_{1}}\left(\frac{s^{4}}{s^{2}-K s+Q^{2}} v^{\prime \prime}(1) \omega(s)-s^{2} f(v(1) \omega(s))-s^{2} g(s)\right) d s d \tau \\
\text { for } r \leq r_{1} \\
0 \quad \text { for } r \geq r_{1},
\end{array}\right.
$$

$u_{1} \equiv 0$. Here $v(t)$ is fixed function which satisfies the hypothesis $(H 1),(H 2)$ and

$$
\text { (H4) }\left\{\begin{array}{l}
\min _{t \in[0,1]} \frac{v^{\prime \prime}(t)}{v(t)} \geq \frac{a}{4 A^{4}}, \quad \max _{t \in[0,1]} \frac{v^{\prime \prime}(t)}{v(t)} \leq \frac{2 a}{A^{2}} ; \\
\lim _{t \longrightarrow 0}\left[v^{\prime \prime}(t)-\frac{a}{4 A^{4}} v(t)\right]=+0, \quad v^{\prime \prime}(t)-\frac{a}{4 A^{4}} v(t) \geq 0 \quad \text { for } \quad t \in[0,1] .
\end{array}\right.
$$

In section 4 we will prove that the equation $\left(1^{\prime \prime}\right)$ has unique nontrivial solution $\omega(r)$ for which $\omega(r) \in \mathcal{C}^{2}\left[0, r_{1}\right], \omega(r) \in \dot{B}_{p, q}^{\gamma}\left[0, r_{1}\right],|\omega(r)| \leq \frac{2}{A B}$ for $r \in\left[0, r_{1}\right], \omega(r) \geq \frac{1}{A^{2}}$ for $r \in\left[\frac{1}{\alpha}, \frac{1}{\beta}\right], \omega\left(r_{1}\right)=\omega^{\prime}\left(r_{1}\right)=\omega^{\prime \prime}\left(r_{1}\right)=0$.

Example.

1) There exists function $v(t)$ for which (H1)-(H3) are hold. Really, let us consider the function

$$
v(t)=\frac{(t-1)^{2}+\frac{4 A^{4}}{a}-1}{\frac{A^{3}}{a}},
$$

where the constants $A$ and $a$ satisfy the conditions $A>1, \frac{A^{2}}{a}>1$. Then

1) $v(t) \in \mathcal{C}^{3}[0, \infty)$ and $v(t)>0$ for all $t \in[0,1]$, i.e. (H1) is hold.

2)

$$
\begin{aligned}
& v^{\prime}(t)=\frac{2(t-1)}{\frac{A^{3}}{2^{a}}}, \quad v^{\prime}(1)=0, \\
& v^{\prime \prime}(t)=\frac{\frac{A}{3}^{3}}{\frac{A^{\prime}}{a}} \quad \forall \quad t \in[0,1], \\
& v^{\prime \prime \prime}(t)=0, \quad v^{\prime \prime \prime}(1)=0,
\end{aligned}
$$

consequently (H2) is hold. On the other hand we have

$$
\frac{v^{\prime \prime}(t)}{v(t)}=\frac{2}{(t-1)^{2}+\frac{4 A^{4}}{a}-1} .
$$


From here

i.e. (H3) is hold.

$$
\begin{aligned}
& \min _{t \in[0,1]} \frac{v^{\prime \prime}(t)}{v(t)} \geq \frac{a}{2 A^{4}} \\
& \max _{t \in[0,1]} \frac{v^{\prime \prime}(t)}{v(t)} \leq \frac{2 a}{A^{2}} \\
& v^{\prime \prime}(t)-\frac{a}{2 A^{4}} v(t)=\frac{1}{\frac{2 A^{7}}{a^{2}}}(2-t) t \\
& \lim _{t \longrightarrow 0}\left[v^{\prime \prime}(t)-\frac{a}{2 A^{4}} v(t)\right]=+0
\end{aligned}
$$

2) The function

$$
v(t)=\frac{(t-1)^{2}+\frac{8 A^{4}}{a}-1}{\frac{A^{3}}{a}},
$$

satisfies the hypothesis (H1), (H2) and (H4).

Remark. Here we will use the following definition of the $\dot{B}_{p, q}^{\gamma}(M)$-norm $(\gamma \in$ $(0,1), p>1, q \geq 1)($ see $[3$, p.94, def. 2], [1])

$$
\|u\|_{\dot{B}_{p, q}^{\gamma}(M)}=\left(\int_{0}^{2} h^{-1-q \gamma}\left\|\Delta_{h} u\right\|_{L^{p}(M)}^{q} d h\right)^{\frac{1}{q}}
$$

where

$$
\Delta_{h} u=u(x+h)-u(x) .
$$

Lemma 1.Let $u(x) \in \mathcal{C}^{2}\left(\left[0, r_{1}\right]\right), u(x)=0$ for $x \geq r_{1}, 0<r_{1}<1$. Then for $\gamma \in(0,1), p>1, q \geq 1$ we have

$$
C|| u\left\|_{\dot{B}_{p, q}^{\gamma}\left(\left[0, r_{1}\right]\right)} \geq\right\| u \|_{L^{p}\left(\left[0, r_{1}\right]\right)} .
$$

Proof. We have

$$
\begin{gathered}
\|u\|_{\dot{B}_{p, q}^{\gamma}\left(\left[0, r_{1}\right]\right)}^{q}=\int_{0}^{2} h^{-1-q \gamma}\left\|\Delta_{h} u\right\|_{L^{p}\left(\left[0, r_{1}\right]\right)}^{q} d h= \\
=\int_{0}^{2} h^{-1-q \gamma}\|u(x+h)-u(x)\|_{L^{p}\left(\left[0, r_{1}\right]\right)}^{q} d h \geq \\
\geq \int_{1}^{2} h^{-1-q \gamma}\|u(x+h)-u(x)\|_{L^{p}\left(\left[0, r_{1}\right]\right)}^{q} d h= \\
=\int_{1}^{2} h^{-1-q \gamma}\|u(x)\|_{L^{p}\left(\left[0, r_{1}\right]\right)}^{q} d h= \\
=\|u(x)\|_{L^{p}\left(\left[0, r_{1}\right]\right)}^{q} \int_{1}^{2} h^{-1-q \gamma} d h= \\
=\|u(x)\|_{L^{p}\left(\left[0, r_{1}\right]\right)}^{q} \frac{2^{q \gamma}-1}{q \gamma 2^{q \gamma}},
\end{gathered}
$$

i.e.

from where we get

$$
\|u\|_{\dot{B}_{p, q}^{\gamma}\left(\left[0, r_{1}\right]\right)}^{q} \geq \frac{2^{q \gamma}-1}{q \gamma 2^{q \gamma}}\|u(x)\|_{L^{p}\left(\left[0, r_{1}\right]\right)}^{q},
$$

$$
C\|u\|_{\dot{B}_{p, q}^{\gamma}\left(\left[0, r_{1}\right]\right)} \geq\|u(x)\|_{L^{p}\left(\left[0, r_{1}\right]\right)} \cdot \bullet
$$

Remark. We note that from i6) we have $g(r)=r^{2}-K r+Q^{2}>0$ for $r \in\left[0, r_{1}\right]$, $g(r)$ is decrease function for $r \in\left[0, r_{1}\right]$. Also (for $r \in\left[0, r_{1}\right]$ ) we have

$$
\frac{r^{2}}{r^{2}-K r+Q^{2}} \leq \frac{1}{1-K+Q^{2}}
$$


Really, let

$$
\epsilon=\sqrt{1-K+Q^{2}}
$$

Then, if $\tilde{r}=\frac{K-\sqrt{K^{2}-4 Q^{2}}}{2}$, we have $r_{1}=\tilde{r}-\epsilon$. We note that

$$
\frac{r^{2}}{r^{2}-K r+Q^{2}}
$$

is increase function for $r \in\left[0, r_{1}\right]$. Therefore

$$
\frac{r^{2}}{r^{2}-K r+Q^{2}} \leq \frac{r_{1}^{2}}{r_{1}^{2}-K r_{1}+Q^{2}} \leq \frac{1}{\epsilon^{2}}=\frac{1}{1-K+Q^{2}}
$$

Also we have

$$
\frac{1}{r^{2}-K r+Q^{2}} \leq \frac{1}{1-K+Q^{2}}
$$

for $r \in\left[0, r_{1}\right]$.

Proposition 2.1. Let $g(r) \equiv 0, f \in \mathcal{C}(\mathcal{R})$. If for every fixed $t \in[0,1]$ $u(t, r)=v(t) \omega(r)$ satisfies the integral equation

$\left(1^{\star}\right) u(t, r)=\int_{r}^{r_{1}} \frac{1}{\tau^{2}-K \tau+Q^{2}} \int_{r}^{r_{1}}\left(\frac{s^{4}}{s^{2}-K s+Q^{2}} \frac{v^{\prime \prime}(t)}{v(t)} u(t, s)-s^{2} f(u)\right) d s d \tau$

then $u(t, r)=v(t) \omega(r)$ satisfies the equation (1) for every fixed $t \in[0,1]$. Here $v(t)$ is function which satisfies the hypothesis $(\mathrm{H} 1)-(\mathrm{H} 3)$ and $\omega(r) \in \mathcal{C}^{2}(\mathcal{R})$.

Proof. Let $t \in[0,1]$ is fixed. Let also $u(t, r)=v(t) \omega(r)$ satisfies the integral equation $\left(1^{\star}\right)$ for every fixed $t \in[0,1]$. Then for fixed $t \in[0,1]$ we have

$$
\begin{aligned}
& u_{r}(t, r)=\frac{1}{r^{2}-K r+Q^{2}} \int_{r_{1}}^{r}\left(\frac{s^{4}}{s^{2}-K s+Q^{2}} \frac{v^{\prime \prime}(t)}{v(t)} u(t, s)-s^{2} f(u)\right) d s, \\
& \left(r^{2}-K r+Q^{2}\right) u_{r}(t, r)=\int_{r_{1}}^{r}\left(\frac{s^{4}}{s^{2}-K s+Q^{2}} \frac{v^{\prime \prime}(t)}{v(t)} u(t, s)-s^{2} f(u)\right) d s, \\
& \partial_{r}\left(\left(r^{2}-K r+Q^{2}\right) u_{r}(t, r)\right)=\frac{r^{4}}{r^{2}-K r+Q^{2}} \frac{v^{\prime \prime}(t)}{v(t)} u(t, r)-r^{2} f(u),
\end{aligned}
$$

Since for every fixed $t \in[0,1]$ we have

$$
u_{t t}(t, r)=v^{\prime \prime}(t) \omega(r)
$$

we have

$$
\partial_{r}\left(\left(r^{2}-K r+Q^{2}\right) u_{r}(t, r)\right)=\frac{r^{4}}{r^{2}-K r+Q^{2}} u_{t t}(t, r)-r^{2} f(u)
$$

for every fixed $t \in[0,1]$. From here for every fixed $t \in[0,1]$ we have

$$
\frac{r^{2}}{r^{2}-K r+Q^{2}} u_{t t}(t, r)-\frac{1}{r^{2}} \partial_{r}\left(\left(r^{2}-K r+Q^{2}\right) u_{r}\right)=f(u),
$$

i.e. for every fixed $t \in[0,1]$ if $u(t, r)=v(t) \omega(r)$, where $v(t)$ is function which satisfies the hypothesis (H1)-(H3) and $\omega(r) \in \mathcal{C}^{2}(\mathcal{R})$, satisfies the integral equation $\left(1^{\star}\right)$ we have that $u(t, r)=v(t) \omega(r)$ satisfies the equation (1) for every fixed $t \in$ $[0,1]$.

Proposition 2.2. Let $g(r) \equiv 0, f \in \mathcal{C}(\mathcal{R})$. If for every fixed $t \in[0,1]$ $u(t, r)=v(t) \omega(r)$, where $v(t)$ is function which satisfies the hypothesis (H1)-(H3) and $\omega(r) \in \mathcal{C}^{2}(\mathcal{R}), \omega\left(r_{1}\right)=\omega^{\prime}\left(r_{1}\right)=0$, satisfies the equation (1) then $u(t, r)=$ $v(t) \omega(r)$ satisfies the equation $\left(1^{\star}\right)$ for every fixed $t \in[0,1]$. 
Proof. Let $t \in[0,1]$ is fixed. Let also $u(t, r)=v(t) \omega(r)$, where $v(t)$ is function which satisfies the hypothesis $(\mathrm{H} 1)-(\mathrm{H} 3)$ and $\omega(r) \in \mathcal{C}^{2}(\mathcal{R})$, satisfies the equation (1). Then

$$
\begin{aligned}
& \frac{r^{2}}{r^{2}-K r+Q^{2}} u_{t t}(t, r)-\frac{1}{r^{2}} \partial_{r}\left(\left(r^{2}-K r+Q^{2}\right) u_{r}\right)=f(u), \\
& \partial_{r}\left(\left(r^{2}-K r+Q^{2}\right) u_{r}(t, r)\right)=\frac{r^{4}}{r^{2}-K r+Q^{2}} u_{t t}(t, r)-r^{2} f(u) .
\end{aligned}
$$

Now we integrate the last equation with respect the variable $r$ and we use that $\omega^{\prime}\left(r_{1}\right)=0, u_{r}\left(t, r_{1}\right)=0$ for every fixed $t \in[0,1]$

$$
\left(r^{2}-K r+Q^{2}\right) u_{r}(t, r)=\int_{r_{1}}^{r}\left(\frac{s^{4}}{s^{2}-K s+Q^{2}} u_{t t}(t, s)-s^{2} f(u)\right) d s .
$$

Again we integrate with respect the variable $r$ and we use that $\omega\left(r_{1}\right)=0, u\left(t, r_{1}\right)=$ 0 for every fixed $t \in[0,1]$. Then we get

$$
u(t, r)=\int_{r}^{r_{1}} \frac{1}{\tau^{2}-K \tau+Q^{2}} \int_{\tau}^{r_{1}}\left(\frac{s^{4}}{s^{2}-K s+Q^{2}} u_{t t}(t, s)-s^{2} f(u)\right) d s d \tau
$$

for every fixed $t \in[0,1]$. Since for every fixed $t \in[0,1]$ we have

$$
u_{t t}(t, r)=v^{\prime \prime}(t) \omega(r)
$$

we get

$$
u(t, r)=\int_{r}^{r_{1}} \frac{1}{\tau^{2}-K \tau+Q^{2}} \int_{\tau}^{r_{1}}\left(\frac{s^{4}}{s^{2}-K s+Q^{2}} \frac{v^{\prime \prime}(t)}{v(t)} u(t, s)-s^{2} f(u)\right) d s d \tau
$$

for every fixed $t \in[0,1]$. Therefore, if for every fixed $t \in[0,1] u(t, r)=v(t) \omega(r)$, where $v(t)$ is function which satisfies the hypothesis $(\mathrm{H} 1)-(\mathrm{H} 3)$ and $\omega(r) \in \mathcal{C}^{2}(\mathcal{R})$, $\omega\left(r_{1}\right)=\omega^{\prime}\left(r_{1}\right)=0$, satisfies the equation (1) then $u(t, r)=v(t) \omega(r)$ satisfies the equation $\left(1^{\star}\right)$ for every fixed $t \in[0,1]$.

Proposition 2.3. Let $g(r) \in \mathcal{C}(\mathcal{R}), f \in \mathcal{C}(\mathcal{R})$. If for every fixed $t \in[0,1]$ $u(t, r)=v(t) \omega(r)$ satisfies the integral equation $\left(1^{\star \star}\right)$

$u(t, r)=\int_{r}^{r_{1}} \frac{1}{\tau^{2}-K \tau+Q^{2}} \int_{r}^{r_{1}}\left(\frac{s^{4}}{s^{2}-K s+Q^{2}} \frac{v^{\prime \prime}(t)}{v(t)} u(t, s)-s^{2} f(u)-s^{2} g(s)\right) d s d \tau$

then $u(t, r)=v(t) \omega(r)$ satisfies the equation (1) for every fixed $t \in[0,1]$. Here $v(t)$ is function which satisfies the hypothesis $(\mathrm{H} 1),(\mathrm{H} 2),(\mathrm{H} 4)$ and $\omega(r) \in \mathcal{C}^{2}(\mathcal{R})$.

Proof. Let $t \in[0,1]$ is fixed. Let also $u(t, r)=v(t) \omega(r)$ satisfies the integral equation $\left(1^{\star \star}\right)$ for every fixed $t \in[0,1]$. Then for fixed $t \in[0,1]$ we have

$$
\begin{aligned}
& u_{r}(t, r)=\frac{1}{r^{2}-K r+Q^{2}} \int_{r_{1}}^{r}\left(\frac{s^{4}}{s^{2}-K s+Q^{2}} \frac{v^{\prime \prime}(t)}{v(t)} u(t, s)-s^{2} f(u)-s^{2} g(s)\right) d s, \\
& \left(r^{2}-K r+Q^{2}\right) u_{r}(t, r)=\int_{r_{1}}^{r}\left(\frac{s^{4}}{s^{2}-K s+Q^{2}} \frac{v^{\prime \prime}(t)}{v(t)} u(t, s)-s^{2} f(u)-s^{2} g(s)\right) d s, \\
& \partial_{r}\left(\left(r^{2}-K r+Q^{2}\right) u_{r}(t, r)\right)=\frac{r^{4}}{r^{2}-K r+Q^{2}} \frac{v^{\prime \prime}(t)}{v(t)} u(t, r)-r^{2} f(u)-r^{2} g(r),
\end{aligned}
$$

Since for every fixed $t \in[0,1]$ we have

$$
u_{t t}(t, r)=v^{\prime \prime}(t) \omega(r)
$$

we have

$$
\partial_{r}\left(\left(r^{2}-K r+Q^{2}\right) u_{r}(t, r)\right)=\frac{r^{4}}{r^{2}-K r+Q^{2}} u_{t t}(t, r)-r^{2} f(u)-r^{2} g(r)
$$


for every fixed $t \in[0,1]$. From here for every fixed $t \in[0,1]$ we have

$$
\frac{r^{2}}{r^{2}-K r+Q^{2}} u_{t t}(t, r)-\frac{1}{r^{2}} \partial_{r}\left(\left(r^{2}-K r+Q^{2}\right) u_{r}\right)=f(u)+g(r),
$$

i.e. for every fixed $t \in[0,1]$ if $u(t, r)=v(t) \omega(r)$, where $v(t)$ is function which satisfies the hypothesis (H1), (H2),(H4) and $\omega(r) \in \mathcal{C}^{2}(\mathcal{R})$, satisfies the integral equation $\left(1^{\star \star}\right)$ we have that $u(t, r)=v(t) \omega(r)$ satisfies the equation (1) for every fixed $t \in[0,1]$.

Proposition 2.4. Let $g(r) \in \mathcal{C}(\mathcal{R}), f \in \mathcal{C}(\mathcal{R})$. If for every fixed $t \in[0,1]$ $u(t, r)=v(t) \omega(r)$, where $v(t)$ is function which satisfies the hypothesis (H1), $(\mathrm{H} 2),(\mathrm{H} 4)$ and $\omega(r) \in \mathcal{C}^{2}(\mathcal{R}), \omega\left(r_{1}\right)=\omega^{\prime}\left(r_{1}\right)=0$, satisfies the equation (1) then $u(t, r)=v(t) \omega(r)$ satisfies the equation $\left(1^{\star \star}\right)$ for every fixed $t \in[0,1]$.

Proof. Let $t \in[0,1]$ is fixed. Let also $u(t, r)=v(t) \omega(r)$, where $v(t)$ is function which satisfies the hypothesis (H1), (H2), (H4) and $\omega(r) \in \mathcal{C}^{2}(\mathcal{R}), \omega\left(r_{1}\right)=\omega^{\prime}\left(r_{1}\right)=$ 0 , satisfies the equation (1). Then

$$
\begin{aligned}
& \frac{r^{2}}{r^{2}-K r+Q^{2}} u_{t t}(t, r)-\frac{1}{r^{2}} \partial_{r}\left(\left(r^{2}-K r+Q^{2}\right) u_{r}\right)=f(u)+g(r), \\
& \partial_{r}\left(\left(r^{2}-K r+Q^{2}\right) u_{r}(t, r)\right)=\frac{r^{4}}{r^{2}-K r+Q^{2}} u_{t t}(t, r)-r^{2} f(u)-r^{2} g(r) .
\end{aligned}
$$

Now we integrate the last equation with respect the variable $r$ and we use that $\omega^{\prime}\left(r_{1}\right)=0, u_{r}\left(t, r_{1}\right)=0$ for every fixed $t \in[0,1]$,

$$
\left(r^{2}-K r+Q^{2}\right) u_{r}(t, r)=\int_{r_{1}}^{r}\left(\frac{s^{4}}{s^{2}-K s+Q^{2}} u_{t t}(t, s)-s^{2} f(u)-s^{2} g(s)\right) d s
$$

for every fixed $t \in[0,1]$. Again we integrate with respect the variable $r$ and we use that $\omega\left(r_{1}\right)=0, u\left(t, r_{1}\right)=0$ for every fixed $t \in[0,1]$. Then we get

$$
u(t, r)=\int_{r}^{r_{1}} \frac{1}{\tau^{2}-K \tau+Q^{2}} \int_{\tau}^{r_{1}}\left(\frac{s^{4}}{s^{2}-K s+Q^{2}} u_{t t}(t, s)-s^{2} f(u)-s^{2} g(s)\right) d s d \tau
$$

for every fixed $t \in[0,1]$. Since for every fixed $t \in[0,1]$ we have

$$
u_{t t}(t, r)=v^{\prime \prime}(t) \omega(r)
$$

we get

$u(t, r)=\int_{r}^{r_{1}} \frac{1}{\tau^{2}-K \tau+Q^{2}} \int_{\tau}^{r_{1}}\left(\frac{s^{4}}{s^{2}-K s+Q^{2}} \frac{v^{\prime \prime}(t)}{v(t)} u(t, s)-s^{2} f(u)-s^{2} g(s)\right) d s d \tau$

for every fixed $t \in[0,1]$. Therefore, if for every fixed $t \in[0,1] u(t, r)=v(t) \omega(r)$, where $v(t)$ is function which satisfies the hypothesis (H1), (H2), (H4) and $\omega(r) \in$ $\mathcal{C}^{2}(\mathcal{R}), \omega\left(r_{1}\right)=\omega^{\prime}\left(r_{1}\right)=0$, satisfies the equation (1) then $u(t, r)=v(t) \omega(r)$ satisfies the equation $\left(1^{\star \star}\right)$ for every fixed $t \in[0,1]$.

\section{Proof of Theorem 1}

3.1. Local existence of nontrivial solutions of homogeneous Cauchy problem (1), (2). Let $v(t)$ is fixed function which satisfies the hypothesis $(H 1)-$ (H3).

In this section we will prove that the homogeneous Cauchy problem (1), (2) has nontrivial solution in the form

$$
u(t, r)=\left\{\begin{array}{l}
v(t) \omega(r) \quad \text { for } \quad r \leq r_{1}, \quad t \in[0,1] \\
0 \text { for } \quad r \geq r_{1}, \quad t \in[0,1] .
\end{array}\right.
$$


Let us consider the integral equation

$(\star) \quad u(t, r)=\left\{\begin{array}{c}\int_{r}^{r_{1}} \frac{1}{\tau^{2}-K \tau+Q^{2}} \int_{\tau}^{r_{1}}\left(\frac{s^{4}}{s^{2}-K s+Q^{2}} \frac{v^{\prime \prime}(t)}{v(t)} u(t, s)-s^{2} f(u(t, s))\right) d s d \tau, \\ 0 \leq r \leq r_{1}, \quad t \in[0,1], \\ 0 \quad \text { for } \quad r \geq r_{1}, \quad t \in[0,1],\end{array}\right.$

where $u(t, r)=v(t) \omega(r)$.

Theorem 3.1. Let $v(t)$ is fixed function which satisfies the hypothesis (H1)(H3). Let also $p>1, q \in[1, \infty)$ and $\gamma \in(0,1)$ are fixed and the positive constants $A, a, b, B, Q, K, \alpha>\beta>1$ satisfy the conditions i1)-i5) and $f \in \mathcal{C}^{1}\left(\mathcal{R}^{1}\right)$, $f(0)=0, a|u| \leq f^{\prime}(u) \leq b|u|$. Then the equation $(\star)$ has unique nontrivial solution $u(t, r)=v(t) \omega(r)$ for which $w \in \mathcal{C}^{2}\left[0, r_{1}\right], u(t, r)=u_{r}(t, r)=u_{r r}(t, r)=0$ for $r \geq r_{1}, u(t, r) \in \mathcal{C}\left((0,1] \dot{B}_{p, q}^{\gamma}\left[0, r_{1}\right]\right)$, for $r \in\left[\frac{1}{\alpha}, \frac{1}{\beta}\right]$ and $t \in[0,1] u(t, r) \geq \frac{1}{A^{2}}$, for $r \in\left[0, r_{1}\right]$ and $t \in[0,1]|u(t, r)| \leq \frac{2}{A B}$.

Proof. Let $N$ is the set

$$
\begin{gathered}
N=\left\{u(t, r) \in \mathcal{C}\left([0,1] \times\left[0, r_{1}\right]\right) ; u(t, r)=u_{r}(t, r)=u_{r r}(t, r)=0 \quad \text { for } \quad r \geq r_{1}\right. \\
\text { and } t \in[0,1], u(t, r) \in \mathcal{C}\left((0,1] \dot{B}_{p, q}^{\gamma}\left[0, r_{1}\right]\right) ; \\
\text { for } \quad r \in\left[\frac{1}{\alpha}, \frac{1}{\beta}\right] \quad \text { and } t \in[0,1] \quad u(t, r) \geq \frac{1}{A^{2}} ; \quad u(t, r) \geq 0 \quad \text { for } \quad t \in[0,1] \\
\text { and } \left.\quad r \in\left[\frac{1}{\alpha}, r_{1}\right] ; \text { for } r \in\left[0, r_{1}\right] \quad \text { and } \quad t \in[0,1] \quad|u(t, r)| \leq \frac{2}{A B}\right\} .
\end{gathered}
$$

Let also $t \in[0,1]$ is fixed.

We define the operator $R$ as follow

$R(u)=\int_{r}^{r_{1}} \frac{1}{\tau^{2}-K \tau+Q^{2}} \int_{\tau}^{r_{1}}\left(\frac{s^{4}}{s^{2}-K s+Q^{2}} \frac{v^{\prime \prime}(t)}{v(t)} u(t, s)-s^{2} f(u)\right) d s d \tau, 0 \leq r \leq r_{1}$.

First we will show that $R: N \longrightarrow N$. We have

1) $R(u) \in \mathcal{C}^{2}\left(\left[0, r_{1}\right]\right)$ because $u(t, r) \in \mathcal{C}\left(\left[0, r_{1}\right]\right), R(u) \in \mathcal{C}\left([0,1] \times\left[0, r_{1}\right]\right)$, $\left.R(u)\right|_{r=r_{1}}=0$,

$$
\begin{gathered}
\frac{\partial}{\partial r} R(u)=\frac{1}{r^{2}-K r+Q^{2}} \int_{r_{1}}^{r}\left[\frac{s^{4}}{s^{2}-K s+Q^{2}} \frac{v^{\prime \prime}(t)}{v(t)} u-s^{2} f(u)\right] d s,\left.\frac{\partial}{\partial r} R(u)\right|_{r=r_{1}}=0, \\
\frac{\partial^{2}}{\partial r^{2}} R(u)=\frac{K-2 r}{\left(r^{2}-K r+Q^{2}\right)^{2}} \int_{r_{1}}^{r}\left[\frac{s^{4}}{s^{2}-K s+Q^{2}} \frac{v^{\prime \prime}(t)}{v(t)} u-s^{2} f(u)\right] d s+ \\
+\frac{r^{4}}{\left(r^{2}-K r+Q^{2}\right)^{2}} \frac{v^{\prime \prime}(t)}{v(t)} u(t, r)-\frac{r^{2}}{r^{2}-K r+Q^{2}} f(u) .
\end{gathered}
$$

Since $u\left(t, r_{1}\right)=0, f\left(u\left(t, r_{1}\right)\right)=f(0)=0$ we get that

$$
\left.\frac{\partial^{2}}{\partial r^{2}} R(u)\right|_{r=r_{1}}=0 \text {. }
$$

2) For $r \in\left[0, r_{1}\right]$ and $t \in[0,1]$ we have $|u(t, r)| \leq \frac{2}{A B}$. Then

$$
\begin{gathered}
|R(u)|=\left|\int_{r_{1}}^{r} \frac{1}{\tau^{2}-K \tau+Q^{2}} \int_{r_{1}}^{\tau}\left(\frac{s^{4}}{s^{2}-K s+Q^{2}} \frac{v^{\prime \prime}(t)}{v(t)} u-s^{2} f(u)\right) d s d \tau\right| \leq \\
\leq \int_{r_{1}}^{r} \frac{1}{\tau^{2}-K \tau+Q^{2}} \int_{r_{1}}^{\tau}\left(\frac{s^{4}}{s^{2}-K s+Q^{2}} \frac{v^{\prime \prime}(t)}{v(t)}|u|+s^{2}|f(u)|\right) d s d \tau \leq
\end{gathered}
$$


(since $a|u| \leq f^{\prime}(u) \leq b|u|$ and $f(0)=0$ we have $|f(u)| \leq \frac{b}{2}|u|^{2}$ )

$$
\begin{aligned}
\leq & \int_{r_{1}}^{r} \frac{1}{\tau^{2}-K \tau+Q^{2}} \int_{r_{1}}^{\tau}\left(\frac{s^{4}}{s^{2}-K s+Q^{2}} \frac{v^{\prime \prime}(t)}{v(t)}|u|+\frac{b}{2} s^{2}|u|^{2}\right) d s d \tau= \\
& \int_{r_{1}}^{r} \frac{1}{\tau^{2}-K \tau+Q^{2}} \int_{r_{1}}^{\tau}\left(\frac{s^{4}}{s^{2}-K s+Q^{2}} \frac{v^{\prime \prime}(t)}{v(t)}+\frac{b}{2} s^{2}|u|\right)|u| d s d \tau \leq \\
\leq & \frac{2}{A B} \int_{r_{1}}^{r} \frac{1}{\tau^{2}-K \tau+Q^{2}} \int_{r_{1}}^{\tau}\left(\frac{s^{4}}{s^{2}-K s+Q^{2}} \frac{v^{\prime \prime}(t)}{v(t)}+\frac{b}{A B} s^{2}\right) d s d \tau \leq
\end{aligned}
$$

(here we use that $\frac{r^{2}}{r^{2}-K r+Q^{2}} \leq \frac{1}{1-K+Q^{2}}, \frac{1}{r^{2}-K r+Q^{2}} \leq \frac{1}{1-K+Q^{2}}$ for $r \in\left[0, r_{1}\right]$ )

$$
\begin{aligned}
& \leq \frac{2}{A B} \frac{1}{1-K+Q^{2}}\left(\frac{1}{1-K+Q^{2}} \max _{t \in[0,1]} \frac{v^{\prime \prime}(t)}{v(t)}+\frac{b}{A B}\right) \leq \\
& \leq \frac{2}{A B} \frac{1}{1-K+Q^{2}}\left(\frac{1}{1-K+Q^{2}} \frac{2 a}{A^{2}}+\frac{b}{A B}\right) \leq \frac{2}{A B} .
\end{aligned}
$$

(in the last inequality we use i1)). Consequently

$$
|R(u)| \leq \frac{2}{A B} \quad \text { for } \quad r \in\left[0, r_{1}\right], \quad t \in[0,1] .
$$

3) For $r \in\left[\frac{1}{\alpha}, r_{1}\right]$ and $t \in[0,1]$ we have $u(t, r) \geq 0$. Then $a u \leq f^{\prime}(u) \leq b u$ for $r \in\left[\frac{1}{\alpha}, r_{1}\right]$ and $t \in[0,1]$. Since $f(0)=0$ we conclude that $f(u) \leq \frac{b}{2} u^{2}$ for $r \in\left[\frac{1}{\alpha}, r_{1}\right]$ and $t \in[0,1]$. Then for $t \in[0,1]$ and $r \in\left[\frac{1}{\alpha}, r_{1}\right]$ we have

$$
\begin{aligned}
R(u) & =\int_{r}^{r_{1}} \frac{1}{\tau^{2}-K \tau+Q^{2}} \int_{\tau}^{r_{1}}\left(\frac{s^{4}}{s^{2}-K s+Q^{2}} \frac{v^{\prime \prime}(t)}{v(t)} u(t, s)-s^{2} f(u)\right) d s d \tau \geq \\
\geq & \int_{r}^{r_{1}} \frac{1}{\tau^{2}-K \tau+Q^{2}} \int_{\tau}^{r_{1}}\left(\frac{s^{4}}{s^{2}-K s+Q^{2}} \frac{v^{\prime \prime}(t)}{v(t)} u(t, s)-s^{2} \frac{b}{2} u^{2}\right) d s d \tau= \\
& =\int_{r}^{r_{1}} \frac{1}{\tau^{2}-K \tau+Q^{2}} \int_{\tau}^{r_{1}}\left(\frac{s^{4}}{s^{2}-K s+Q^{2}} \frac{v^{\prime \prime}(t)}{v(t)}-s^{2} \frac{b}{2} u\right) u d s d \tau \geq
\end{aligned}
$$

(now we use that $u(t, r) \leq \frac{2}{A B}$ for $r \in\left[\frac{1}{\alpha}, r_{1}\right]$ and $t \in[0,1]$ )

$$
\begin{gathered}
\geq \int_{r}^{r_{1}} \frac{1}{\tau^{2}-K \tau+Q^{2}} \int_{\tau}^{r_{1}}\left(\frac{s^{4}}{s^{2}-K s+Q^{2}} \frac{v^{\prime \prime}(t)}{v(t)}-s^{2} \frac{b}{A B}\right) u d s d \tau \geq \\
\geq \int_{r}^{r_{1}} \frac{1}{\tau^{2}-K \tau+Q^{2}} \int_{\tau}^{r_{1}}\left(\frac{s^{4}}{s^{2}-K s+Q^{2}} \min _{t \in[0,1]} \frac{v^{\prime \prime}(t)}{v(t)}-s^{2} \frac{b}{A B}\right) u d s d \tau \geq
\end{gathered}
$$

(here we use (H3))

$$
\geq \int_{r}^{r_{1}} \frac{1}{\tau^{2}-K \tau+Q^{2}} \int_{\tau}^{r_{1}}\left(\frac{s^{4}}{s^{2}-K s+Q^{2}} \frac{a}{2 A^{4}}-s^{2} \frac{b}{A B}\right) u d s d \tau \geq
$$

(Here we use that for $s \in\left[0, r_{1}\right]$ the function $\frac{s^{2}}{s^{2}-K s+Q^{2}}$ is increase function. Therefore for $s \in\left[\frac{1}{\alpha}, r_{1}\right]$ we have $\left.\frac{s^{4}}{s^{2}-K s+Q^{2}} \geq \frac{1}{\alpha^{2}\left(1-\alpha K+\alpha^{2} Q^{2}\right)}\right)$

$$
\begin{aligned}
& \geq \int_{r}^{r_{1}} \frac{1}{\tau^{2}-K \tau+Q^{2}} \int_{\tau}^{r_{1}}\left(\frac{1}{\alpha^{2}\left(1-\alpha K+\alpha^{2} Q^{2}\right)} \frac{a}{2 A^{4}}-s^{2} \frac{b}{A B}\right) u d s d \tau \geq \\
& \geq \int_{r}^{r_{1}} \frac{1}{\tau^{2}-K \tau+Q^{2}} \int_{\tau}^{r_{1}}\left(\frac{1}{\alpha^{2}\left(1-\alpha K+\alpha^{2} Q^{2}\right)} \frac{a}{2 A^{4}}-r_{1}^{2} \frac{b}{A B}\right) u d s d \tau,
\end{aligned}
$$


i.e.

(4) $\quad R(u) \geq \int_{r}^{r_{1}} \frac{1}{\tau^{2}-K \tau+Q^{2}} \int_{\tau}^{r_{1}}\left(\frac{1}{\alpha^{2}\left(1-\alpha K+\alpha^{2} Q^{2}\right)} \frac{a}{2 A^{4}}-r_{1}^{2} \frac{b}{A B}\right) u d s d \tau$ for $r \in\left[\frac{1}{\alpha}, r_{1}\right]$ and $t \in[0,1]$. From the first inequality of i2) we have

$$
\frac{1}{\alpha^{2}\left(1-\alpha K+\alpha^{2} Q^{2}\right)} \frac{a}{2 A^{4}}-r_{1}^{2} \frac{b}{A B} \geq 0 .
$$

Since $u(t, r) \geq 0$ for $r \in\left[\frac{1}{\alpha}, r_{1}\right], t \in[0,1]$ and $\frac{1}{\tau^{2}-K \tau+Q^{2}}>0$ for $\tau \in\left[0, r_{1}\right]$ from (4) we conclude that $R(u) \geq 0$ for $r \in\left[\frac{1}{\alpha}, r_{1}\right]$ and $t \in[0,1]$.

On the other hand, for $r \in\left[\frac{1}{\alpha}, \frac{1}{\beta}\right]$,

$$
\frac{\partial}{\partial r} R(u)=\frac{1}{r^{2}-K r+Q^{2}} \int_{r}^{r_{1}}\left(s^{2} f(u)-\frac{v^{\prime \prime}(t)}{v(t)} \frac{s^{4}}{s^{2}-K s+Q^{2}} u\right) d s .
$$

Let

$$
l(r)=\int_{r}^{r_{1}}\left(s^{2} f(u)-\frac{v^{\prime \prime}(t)}{v(t)} \frac{s^{4}}{s^{2}-K s+Q^{2}} u\right) d s .
$$

Then

$$
l^{\prime}(r)=-r^{2} f(u)+\frac{v^{\prime \prime}(t)}{v(t)} \frac{r^{4}}{r^{2}-K r+Q^{2}} u .
$$

Then for $r \in\left[\frac{1}{\alpha}, \frac{1}{\beta}\right]$ we have

$$
l^{\prime}(r) \geq \frac{a}{2 A^{4}} \frac{1}{\alpha^{2}\left(1-\alpha K+\alpha^{2} Q^{2}\right)} \frac{1}{A^{2}}-2 r_{1}^{2} \frac{b}{A^{2} B^{2}} \geq 0 .
$$

(see second inequality of i2)). Consequently $l(r)$ is increase function for $r \in\left[\frac{1}{\alpha}, \frac{1}{\beta}\right]$. From here

$$
l(r) \geq \int_{\frac{1}{\alpha}}^{r_{1}}\left(s^{2} f(u)-\frac{v^{\prime \prime}(t)}{v(t)} \frac{s^{4}}{s^{2}-K s+Q^{2}} u\right) d s
$$

for $r \in\left[\frac{1}{\alpha}, \frac{1}{\beta}\right]$. Since $\frac{1}{r^{2}-K r+Q^{2}} \geq 0$ for $r \in\left[\frac{1}{\alpha}, \frac{1}{\beta}\right]$ we have for $r \in\left[\frac{1}{\alpha}, \frac{1}{\beta}\right]$

$$
\begin{aligned}
& \frac{\partial}{\partial r} R(u) \geq \frac{1}{r^{2}-K r+Q^{2}} \int_{\frac{1}{\alpha}}^{r_{1}}\left(s^{2} f(u)-\frac{v^{\prime \prime}(t)}{v(t)} \frac{s^{4}}{s^{2}-K s+Q^{2}} u\right) d s \geq \\
& \geq \frac{1}{r^{2}-K r+Q^{2}}\left(\int_{\frac{1}{\alpha}}^{\frac{1}{\beta}} s^{2} f(u) d s-\int_{0}^{r_{1}} \frac{v^{\prime \prime}(t)}{v(t)} \frac{s^{4}}{s^{2}-K s+Q^{2}} u d s\right) \geq \\
& \quad \geq \frac{a}{2 A^{4}\left(1-\alpha K+\alpha^{2} Q^{2}\right)}\left(\frac{1}{\beta}-\frac{1}{\alpha}\right)-\frac{4 a r_{1}}{A^{3} B\left(1-K+Q^{2}\right)^{2}} \geq 0 .
\end{aligned}
$$

(see third inequality of i2)) Therefore $R(u)$ is increase function for $r \in\left[\frac{1}{\alpha}, \frac{1}{\beta}\right]$. Consequently, for $r \in\left[\frac{1}{\alpha}, \frac{1}{\beta}\right]$,

$$
\begin{aligned}
R(u) \geq & \int_{\frac{1}{\alpha}}^{r_{1}} \frac{1}{\tau^{2}-K \tau+Q^{2}} \int_{\tau}^{r_{1}}\left(\frac{s^{4}}{s^{2}-K s+Q^{2}} \frac{v^{\prime \prime}(t)}{v(t)} u-s^{2} f(u)\right) d s d \tau \geq \\
& \geq \int_{\frac{1}{\alpha}}^{\frac{1}{\beta}} \frac{1}{\tau^{2}-K \tau+Q^{2}} \int_{\frac{1}{\alpha}}^{\frac{1}{\beta}} \frac{s^{4}}{s^{2}-K s+Q^{2}} \frac{v^{\prime \prime}(t)}{v(t)} u d s d \tau-
\end{aligned}
$$




$$
\begin{gathered}
\int_{\frac{1}{\alpha}}^{r_{1}} \frac{1}{\tau^{2}-K \tau+Q^{2}} \int_{\tau}^{r_{1}} s^{2} f(u) d s d \tau \geq \\
\geq\left(\frac{1}{\beta}-\frac{1}{\alpha}\right)^{2} \frac{1}{\left(1-\alpha K+\alpha^{2} Q^{2}\right)^{2}} \frac{a}{2 A^{6}}-r_{1}^{2} \frac{2 b}{\left(1-K+Q^{2}\right) A^{2} B^{2}} \geq \frac{1}{A^{2}} .
\end{gathered}
$$

(in the last inequality we use the fourth inequality of i2)) Consequently, for $r \in$ $\left[\frac{1}{\alpha}, \frac{1}{\beta}\right]$ and $t \in[0,1]$ we have

4)

$$
R(u) \geq \frac{1}{A^{2}}
$$

$$
\begin{gathered}
\left\|\Delta_{h} R(u)\right\|_{L^{p}}^{q}= \\
\left(\int_{0}^{r_{1}}\left(\left|\int_{r}^{r+h} \frac{1}{\tau^{2}-K \tau+Q^{2}} \int_{\tau}^{r_{1}}\left(\frac{s^{4}}{s^{2}-K s+Q^{2}} \frac{v^{\prime \prime}(t)}{v(t)} u(t, s)-s^{2} f(u)\right) d s d \tau\right|\right)^{p} d r\right)^{\frac{q}{p}} \leq \\
\leq\left(\int_{0}^{r_{1}}\left(\int_{r}^{r+h} \frac{1}{\tau^{2}-K \tau+Q^{2}} \int_{\tau}^{r_{1}}\left(\frac{s^{4}}{s^{2}-K s+Q^{2}} \frac{v^{\prime \prime}(t)}{v(t)}|u|+\frac{b}{2}|u|^{2} s^{2}\right) d s d \tau\right)^{p} d r\right)^{\frac{q}{p}} \leq
\end{gathered}
$$

(here we use that for $s \in\left[0, r_{1}\right]$ we have $\frac{s^{4}}{s^{2}-K s+Q^{2}} \leq \frac{1}{1-K+Q^{2}}$, also for $r \in\left[0, r_{1}\right]$ and $t \in[0,1]$ we have $\left.|u(t, r)| \leq \frac{2}{A B}\right)$

$$
\begin{gathered}
\leq\left(\int _ { 0 } ^ { r _ { 1 } } \left(\int _ { r } ^ { r + h } \frac { 1 } { 1 - K + Q ^ { 2 } } \int _ { \tau } ^ { r _ { 1 } } \left(\frac{1}{1-K+Q^{2}} \max _{t \in[0,1]} \frac{v^{\prime \prime}(t)}{v(t)}|u|+\right.\right.\right. \\
\left.\left.\left.+\frac{b}{A B}|u|\right) d s d \tau\right)^{p} d r\right)^{\frac{q}{p}} \leq
\end{gathered}
$$

(here we use (H3))

$$
\begin{gathered}
\leq\left(\int_{0}^{r_{1}}\left(\int_{r}^{r+h} \frac{1}{1-K+Q^{2}} \int_{\tau}^{r_{1}}\left(\frac{1}{1-K+Q^{2}} \frac{2 a}{A^{2}}|u|+\frac{b}{A B}|u|\right) d s d \tau\right)^{p} d r\right)^{\frac{q}{p}} \leq \\
\left.\leq\left(\int_{0}^{r_{1}}\left(\int_{r}^{r+h} \frac{1}{\left(1-K+Q^{2}\right)^{2}} \frac{2 a}{A^{2}} \int_{0}^{r_{1}}|u| d s+\frac{1}{1-K+Q^{2}} \frac{b}{A B} \int_{0}^{r_{1}}|u| d s\right) d \tau\right)^{p} d r\right)^{\frac{q}{p}} \\
\leq h^{q}\left(\frac{1}{\left(1-K+Q^{2}\right)^{2}} \frac{2 a}{A^{2}}\|u\|_{L^{p}}+\frac{1}{1-K+Q^{2}} \frac{b}{A B}\|u\|_{L^{p}}\right)^{q},
\end{gathered}
$$

i.e.

$$
\left\|\Delta_{h} R(u)\right\|_{L^{p}}^{q} \leq h^{q}\left(\frac{1}{\left(1-K+Q^{2}\right)^{2}} \frac{2 a}{A^{2}}\|u\|_{L^{p}}+\frac{1}{1-K+Q^{2}} \frac{b}{A B}\|u\|_{L^{p}}\right)^{q} .
$$

Consequently

$$
\begin{gathered}
\|R(u)\|_{\dot{B}_{p, q}^{\gamma}\left[0, r_{1}\right]}^{q}=\int_{0}^{2} h^{-1-q \gamma}\left\|\Delta_{h} R(u)\right\|_{L^{p}}^{q} d h \leq \\
\leq\left(\frac{1}{\left(1-K+Q^{2}\right)^{2}} \frac{2 a}{A^{2}}\|u\|_{L^{p}}+\frac{b}{A B\left(1-K+Q^{2}\right)}\|u\|_{L^{p}}\right)^{q} \int_{0}^{2} h^{-1+q(1-\gamma)} d h= \\
=\left(\frac{1}{\left(1-K+Q^{2}\right)^{2}} \frac{2 a}{A^{2}}\|u\|_{L^{p}}+\frac{b}{A B\left(1-K+Q^{2}\right)}\|u\|_{L^{p}}\right)^{q} \frac{2^{q(1-\gamma)}}{q(1-\gamma)} .
\end{gathered}
$$

Therefore

$\|R(u)\|_{\dot{B}_{P, q}^{\gamma}\left[0, r_{1}\right]} \leq\left(\frac{1}{\left(1-K+Q^{2}\right)^{2}} \frac{2 a}{A^{2}}\|u\|_{L^{p}}+\frac{b}{A B\left(1-K+Q^{2}\right)}\|u\|_{L^{p}}\right) \frac{2^{(1-\gamma)}}{(q(1-\gamma))^{\frac{1}{q}}}$. 
From lemma 1 we get

$$
\begin{gathered}
\|R(u)\|_{\dot{B}_{p, q}^{\gamma}\left[0, r_{1}\right]} \leq \\
C\left(\frac{1}{\left(1-K+Q^{2}\right)^{2}} \frac{2 a}{A^{2}}\|u\|_{\dot{B}_{p, q}^{\gamma}\left[0, r_{1}\right]}+\frac{b}{A B\left(1-K+Q^{2}\right)}\|u\|_{\dot{B}_{p, q}^{\gamma}\left[0, r_{1}\right]}\right) \frac{2^{1-\gamma}}{(q(1-\gamma))^{\frac{1}{q}}} .
\end{gathered}
$$

From the last inequality, if $u \in \dot{B}_{p, q}^{\gamma}\left[0, r_{1}\right]$ we get $R(u) \in \dot{B}_{p, q}^{\gamma}\left[0, r_{1}\right]$. From 1$\left.), 2\right)$, 3 ), 4) we get $R: N \longrightarrow N$.

Let $u, u_{1} \in N$. Then

$$
\begin{gathered}
\left\|\Delta_{h}\left(R(u)-R\left(u_{1}\right)\right)\right\|_{L^{p}}^{q}= \\
=\left(\int _ { 0 } ^ { r _ { 1 } } \left(\mid \int_{r}^{r+h} \frac{1}{\tau^{2}-K \tau+Q^{2}} \int_{\tau}^{r_{1}}\left(\frac{s^{4}}{s^{2}-K s+Q^{2}} \frac{v^{\prime \prime}(t)}{v(t)}\left(u(t, s)-u_{1}(t, s)\right)-\right.\right.\right. \\
\leq\left(\int _ { 0 } ^ { r _ { 1 } } \left(\int _ { r } ^ { r + h } \frac { 1 } { \tau ^ { 2 } - K \tau + Q ^ { 2 } } \int _ { \tau } ^ { r _ { 1 } } \left(\frac{s^{4}}{s^{2}-K s+Q^{2}} \frac{v^{\prime \prime}(t)}{v(t)}\left|u-u_{1}\right|+\right.\right.\right. \\
\left.\left.\left.\left|f(u)-f\left(u_{1}\right)\right| s^{2}\right) d s d \tau\right)^{p} d r\right)^{\frac{q}{p}} \leq
\end{gathered}
$$

(from the midle point theorem we have $\left|f(u)-f\left(u_{1}\right)\right|=\left|f^{\prime}(\xi)\right|\left|u-u_{1}\right|$, also we have $\left|f^{\prime}(\xi)\right| \leq b|\xi| \leq b \max \left\{|u|,\left|u_{1}\right|\right\} \leq \frac{2 b}{A B}$, therefore $\left.\left|f(u)-f\left(u_{1}\right)\right| \leq \frac{2 b}{A B}\left|u-u_{1}\right|\right)$

$$
\begin{gathered}
\leq\left(\int _ { 0 } ^ { r _ { 1 } } \left(\int _ { r } ^ { r + h } \frac { 1 } { \tau ^ { 2 } - K \tau + Q ^ { 2 } } \int _ { \tau } ^ { r _ { 1 } } \left(\frac{s^{4}}{s^{2}-K s+Q^{2}} \frac{v^{\prime \prime}(t)}{v(t)}\left|u-u_{1}\right|+\right.\right.\right. \\
\left.\left.\left.\frac{2 b}{A B}\left|u-u_{1}\right| s^{2}\right) d s d \tau\right)^{p} d r\right)^{\frac{q}{p}} \leq
\end{gathered}
$$

(here we use that for $s \in\left(0, r_{1}\right)$ we have $\frac{s^{4}}{s^{2}-K s+Q^{2}} \leq \frac{1}{1-K+Q^{2}}$, also for $r \in\left[0, r_{1}\right]$ we have $\left.\frac{1}{r^{2}-K r+Q^{2}} \leq \frac{1}{1-K+Q^{2}}\right)$

(here we use (H3))

$$
\begin{gathered}
\leq\left(\int _ { 0 } ^ { r _ { 1 } } \left(\int _ { r } ^ { r + h } \frac { 1 } { 1 - K + Q ^ { 2 } } \int _ { \tau } ^ { r _ { 1 } } \left(\frac{1}{1-K+Q^{2}} \max _{t \in[0,1]} \frac{v^{\prime \prime}(t)}{v(t)}\left|u-u_{1}\right|+\right.\right.\right. \\
\left.\left.\left.+\frac{2 b}{A B}\left|u-u_{1}\right|\right) d s d \tau\right)^{p} d r\right)^{\frac{q}{p}} \leq
\end{gathered}
$$

$$
\begin{gathered}
\leq\left(\int _ { 0 } ^ { r _ { 1 } } \left(\int _ { r } ^ { r + h } \frac { 1 } { 1 - K + Q ^ { 2 } } \int _ { \tau } ^ { r _ { 1 } } \left(\frac{1}{1-K+Q^{2}} \frac{2 a}{A^{2}}\left|u-u_{1}\right|+\right.\right.\right. \\
\left.\left.\left.\frac{2 b}{A B}\left|u-u_{1}\right|\right) d s d \tau\right)^{p} d r\right)^{\frac{q}{p}} \leq \\
\leq\left(\int _ { 0 } ^ { r _ { 1 } } \left(\int_{r}^{r+h} \frac{1}{\left(1-K+Q^{2}\right)^{2}} \frac{2 a}{A^{2}} \int_{0}^{r_{1}}\left|u-u_{1}\right| d s\right.\right. \\
\left.\left.\left.+\frac{1}{1-K+Q^{2}} \frac{2 b}{A B} \int_{0}^{r_{1}}\left|u-u_{1}\right| d s\right) d \tau\right)^{p} d r\right)^{\frac{q}{p}} \leq \\
\leq h^{q}\left(\frac{1}{\left(1-K+Q^{2}\right)^{2}} \frac{2 a}{A^{2}}\left\|u-u_{1}\right\|_{L^{p}}+\frac{1}{1-K+Q^{2}} \frac{2 b}{A B}\left\|u-u_{1}\right\|_{L^{p}}\right)^{q},
\end{gathered}
$$

i.e.

$$
\left\|\Delta_{h}\left(R(u)-R\left(u_{1}\right)\right)\right\|_{L^{p}}^{q} \leq h^{q}\left(\frac{1}{\left(1-K+Q^{2}\right)^{2}} \frac{2 a}{A^{2}}\left\|u-u_{1}\right\|_{L^{p}}+\right.
$$


Consequently

$$
\left.\frac{1}{1-K+Q^{2}} \frac{2 b}{A B}\left\|u-u_{1}\right\|_{L^{p}}\right)^{q} .
$$

$$
\begin{gathered}
\left\|R(u)-R\left(u_{1}\right)\right\|_{\dot{B}_{p, q}^{\gamma}\left[0, r_{1}\right]}^{q}=\int_{0}^{2} h^{-1-q \gamma}\left\|\Delta_{h}\left(R(u)-R\left(u_{1}\right)\right)\right\|_{L^{p}}^{q} d h \leq \\
\leq\left(\frac{1}{\left(1-K+Q^{2}\right)^{2}} \frac{2 a}{A^{2}}\left\|u-u_{1}\right\|_{L^{p}}+\right. \\
\left.\frac{2 b}{A B\left(1-K+Q^{2}\right)}\left\|u-u_{1}\right\|_{L^{p}}\right)^{q} \int_{0}^{2} h^{-1+q(1-\gamma)} d h= \\
=\left(\frac{1}{\left(1-K+Q^{2}\right)^{2}} \frac{2 a}{A^{2}}\left\|u-u_{1}\right\|_{L^{p}}+\frac{2 b}{A B\left(1-K+Q^{2}\right)}\left\|u-u_{1}\right\|_{L^{p}}\right)^{q} \frac{2^{q(1-\gamma)}}{q(1-\gamma)} .
\end{gathered}
$$

Therefore

$$
\begin{gathered}
\left\|R(u)-R\left(u_{1}\right)\right\|_{\dot{B}_{p, q}^{\gamma}\left[0, r_{1}\right]}^{q} \leq \\
\leq\left(\frac{1}{\left(1-K+Q^{2}\right)^{2}} \frac{2 a}{A^{2}}\left\|u-u_{1}\right\|_{L^{p}}+\frac{2 b}{A B\left(1-K+Q^{2}\right)}\left\|u-u_{1}\right\|_{L^{p}}\right)^{q} \frac{2^{q(1-\gamma)}}{(q(1-\gamma))} .
\end{gathered}
$$

From lemma 1 we get

$$
\begin{gathered}
\left\|R(u)-R\left(u_{1}\right)\right\|_{\dot{B}_{p, q}^{\gamma}\left[0, r_{1}\right]} \leq \\
\leq C\left(\frac{1}{\left(1-K+Q^{2}\right)^{2}} \frac{2 a}{A^{2}}\left\|u-u_{1}\right\|_{\dot{B}_{p, q}^{\gamma}\left[0, r_{1}\right]}+\right. \\
\left.\frac{2 b}{A B\left(1-K+Q^{2}\right)}\left\|u-u_{1}\right\|_{\dot{B}_{p, q}^{\gamma}\left[0, r_{1}\right]}\right) \frac{2^{1-\gamma}}{(q(1-\gamma))^{\frac{1}{q}}} .
\end{gathered}
$$

From i3) we have

$$
C\left(\frac{1}{\left(1-K+Q^{2}\right)^{2}} \frac{2 a}{A^{2}}+\frac{2 b}{A B\left(1-K+Q^{2}\right)}\right) \frac{2^{1-\gamma}}{(q(1-\gamma))^{\frac{1}{q}}}<1 .
$$

therefore

$$
\left\|R(u)-R\left(u_{1}\right)\right\|_{\dot{B}_{p, q}^{\gamma}\left[0, r_{1}\right]}<\left\|u-u_{1}\right\|_{\dot{B}_{p, q}^{\gamma}\left[0, r_{1}\right]} .
$$

Consequently the operator $R: N \longrightarrow N$ is contractive operator. We note that $N$ is closed subset of $\mathcal{C}\left((0,1] \dot{B}_{p, q}^{\gamma}\left[0, r_{1}\right]\right)$ (for the proof see lemma 5.1 in the appendix of this paper). Therefore the equation $(\star)$ has unique nontrivial solution in the set $N$.

Let $\tilde{u}$ is the solution from the theorem 3. 1, i.e $\tilde{u}$ is the solution to the equation $(\star)$. From proposition $2.1 \tilde{u}$ satisfies the equation (1). Then $\tilde{u}$ is solution to the Cauchy problem (1), (2) with initial data

$$
\begin{gathered}
u_{0}=\left\{\begin{array}{c}
\int_{r}^{r_{1}} \frac{1}{\tau^{2}-K \tau+Q^{2}} \int_{\tau}^{r_{1}}\left(\frac{s^{4}}{s^{2}-K s+Q^{2}} v^{\prime \prime}(1) \omega(s)-s^{2} f(v(1) \omega(s))\right) d s d \tau \\
\text { for } r \leq r_{1}, \\
\text { for } r \geq r_{1},
\end{array}\right. \\
u_{1}=\left\{\begin{array}{c}
\int_{r}^{r_{1}} \frac{1}{\tau^{2}-K \tau+Q^{2}} \int_{\tau}^{r_{1}}\left(\frac{s^{4}}{s^{2}-K s+Q^{2}} v^{\prime \prime \prime}(1) \omega(s)-s^{2} f^{\prime}(u) v^{\prime}(1) \omega(s)\right) d s d \tau=0 \\
\text { for } \quad \text { for } r \geq r_{1},
\end{array}\right.
\end{gathered}
$$

$u_{0} \in \dot{B}_{p, q}^{\gamma}\left(\mathcal{R}^{+}\right), u_{1} \in \dot{B}_{p, q}^{\gamma-1}\left(\mathcal{R}^{+}\right), \tilde{u} \in \mathcal{C}\left((0,1] \dot{B}_{p, q}^{\gamma}\left[0, r_{1}\right]\right)$. 
3.2. Blow up of the solutions of the homogeneous Cauchy problem (1), (2). Let $v(t)$ is same function as in Theorem 3.1.

Theorem 3.2. Let $p>1, q \geq 1$ and $\gamma \in(0,1)$ are fixed and the positive constants $a, b, A, B, Q, K, 1<\beta<\alpha$ satisfy the conditions i1)-i5). Let $f \in$ $\mathcal{C}^{1}\left(\mathcal{R}^{1}\right), f(0)=0, a|u| \leq f^{\prime}(u) \leq b|u|$. Then for the solution $\tilde{u}$ to the Cauchy problem (1), (2) we have

$$
\lim _{t \longrightarrow 0}\|\tilde{u}\|_{\dot{B}_{p, q}^{\gamma}\left[0, r_{1}\right]}=\infty \text {. }
$$

Proof. We suppose that $t \in(0,1]$. Then we have

$$
\begin{gathered}
\left\|\Delta_{h} R(\tilde{u})\right\|_{L^{p}}^{q}=\left(\int_{0}^{r_{1}} \mid \int_{r}^{r+h} \frac{1}{\tau^{2}-K \tau+Q^{2}} \int_{\tau}^{r_{1}}\left[\frac{s^{4}}{s^{2}-K s+Q^{2}} \frac{v^{\prime \prime}(t)}{v(t)} \tilde{u}\right.\right. \\
\left.\left.-s^{2} f(\tilde{u})\right]\left.d s d \tau\right|^{p} d r\right)^{\frac{q}{p}}= \\
=\left(\int_{0}^{\frac{1}{\alpha}}\left|\int_{r}^{r+h} \frac{1}{\tau^{2}-K \tau+Q^{2}} \int_{\tau}^{r_{1}}\left[\frac{s^{4}}{s^{2}-K s+Q^{2}} \frac{v^{\prime \prime}(t)}{v(t)} \tilde{u}-s^{2} f(\tilde{u})\right] d s d \tau\right|^{p} d r+\right. \\
\left.+\int_{\frac{1}{\alpha}}^{r_{1}}\left|\int_{r}^{r+h} \frac{1}{\tau^{2}-K \tau+Q^{2}} \int_{\tau}^{r_{1}}\left[\frac{s^{4}}{s^{2}-K s+Q^{2}} \frac{v^{\prime \prime}(t)}{v(t)} \tilde{u}-s^{2} f(\tilde{u})\right] d s d \tau\right|^{p} d r\right)^{\frac{q}{p}} .
\end{gathered}
$$

Let

$$
\begin{aligned}
& I_{1}=\int_{0}^{\frac{1}{\alpha}}\left|\int_{r}^{r+h} \frac{1}{\tau^{2}-K \tau+Q^{2}} \int_{\tau}^{r_{1}}\left[\frac{s^{4}}{s^{2}-K s+Q^{2}} \frac{v^{\prime \prime}(t)}{v(t)} \tilde{u}-s^{2} f(\tilde{u})\right] d s d \tau\right|^{p} d r, \\
& \left.I_{2}=\int_{\frac{1}{\alpha}}^{r_{1}} \mid \int_{r}^{r+h} \frac{1}{\tau^{2}-K \tau+Q^{2}} \int_{\tau}^{r_{1}}\left[\frac{s^{4}}{s^{2}-K s+Q^{2}} \frac{v^{\prime \prime}(t)}{v(t)} \tilde{u}-s^{2} f(\tilde{u})\right)\right]\left.d s d \tau\right|^{p} d r .
\end{aligned}
$$

For $I_{1}$ we have the following estimate

$$
I_{1} \leq C^{p} \frac{1}{\left(1-K+Q^{2}\right)^{p}}\left(\frac{1}{1-K+Q^{2}} \frac{2 a}{A^{2}}+\frac{b}{A B}\right)^{p}\|\tilde{u}\|_{\dot{B}_{p, q}^{\gamma}}^{p} h^{p} .
$$

(in the last inequality we use that $|f(u)| \leq \frac{b}{2}|u|^{2} \leq \frac{b}{A B}|u|$ ) For $I_{2}$ we have

$$
\begin{aligned}
I_{2} & =\int_{\frac{1}{\alpha}}^{r_{1}} \mid \int_{r}^{r+h} \frac{1}{\tau^{2}-K \tau+Q^{2}} \int_{\frac{1}{\alpha}}^{\frac{1}{\beta}}\left[\frac{s^{4}}{s^{2}-K s+Q^{2}} \frac{v^{\prime \prime}(t)}{v(t)} \tilde{u}-s^{2} f(\tilde{u})\right] d s d \tau+ \\
& +\left.\int_{r}^{r+h} \frac{1}{\tau^{2}-K \tau+Q^{2}} \int_{\frac{1}{\beta}}^{r_{1}}\left[\frac{s^{4}}{s^{2}-K s+Q^{2}} \frac{v^{\prime \prime}(t)}{v(t)} \tilde{u}-s^{2} f(\tilde{u})\right] d s d \tau\right|^{p} d r \leq \\
& \leq \int_{\frac{1}{\alpha}}^{r_{1}}\left(\int_{r}^{r+h} \frac{1}{\tau^{2}-K \tau+Q^{2}} \int_{\frac{1}{\alpha}}^{\frac{1}{\beta}}\left[\frac{s^{4}}{s^{2}-K s+Q^{2}} \frac{v^{\prime \prime}(t)}{v(t)} \tilde{u}-s^{2} f(\tilde{u})\right] d s d \tau+\right. \\
& \left.+\left|\int_{r}^{r+h} \frac{1}{\tau^{2}-K \tau+Q^{2}} \int_{\frac{1}{\beta}}^{r_{1}}\left[\frac{s^{4}}{s^{2}-K s+Q^{2}} \frac{v^{\prime \prime}(t)}{v(t)} \tilde{u}-s^{2} f(\tilde{u})\right] d s d \tau\right|\right)^{p} d r .
\end{aligned}
$$

Let

$$
\begin{gathered}
I_{21}=\int_{r}^{r+h} \frac{1}{\tau^{2}-K \tau+Q^{2}} \int_{\frac{1}{\alpha}}^{\frac{1}{\beta}}\left[\frac{s^{4}}{s^{2}-K s+Q^{2}} \frac{v^{\prime \prime}(t)}{v(t)} \tilde{u}-s^{2} f(\tilde{u})\right] d s d \tau, \\
I_{22}=\left|\int_{r}^{r+h} \frac{1}{\tau^{2}-K \tau+Q^{2}} \int_{\frac{1}{\beta}}^{r_{1}}\left[\frac{s^{4}}{s^{2}-K s+Q^{2}} \frac{v^{\prime \prime}(t)}{v(t)} \tilde{u}-s^{2} f(\tilde{u})\right] d s d \tau\right| .
\end{gathered}
$$


Then

$$
I_{2} \leq \int_{\frac{1}{\alpha}}^{r_{1}}\left(I_{21}+I_{22}\right)^{p} d r
$$

For $I_{21}$ we have the following estimate ( here we use that for $r \in\left[\frac{1}{\alpha}, \frac{1}{\beta}\right] \tilde{u} \geq 0$, $f(\tilde{u}) \geq \frac{a}{2} \tilde{u}^{2} \geq \frac{a}{2 A^{2}} \tilde{u}$, therefore $\left.-f(\tilde{u}) \leq-\frac{a}{2 A^{2}} \tilde{u}\right)$

$$
\begin{aligned}
& I_{21} \leq \int_{r}^{r+h} \frac{1}{\tau^{2}-K \tau+Q^{2}} \int_{\frac{1}{\alpha}}^{\frac{1}{\beta}}\left(\frac{s^{4}}{s^{2}-K s+Q^{2}} \frac{v^{\prime \prime}(t)}{v(t)} \tilde{u}-\frac{a}{2 A^{2}} s^{2} \tilde{u}\right) d s d \tau= \\
& =\int_{r}^{r+h} \frac{1}{\tau^{2}-K \tau+Q^{2}} \int_{\frac{1}{\alpha}}^{\frac{1}{\beta}}\left(\frac{s^{4}}{s^{2}-K s+Q^{2}} \frac{v^{\prime \prime}(t)}{v(t)} \frac{A^{2} \tilde{u}}{A^{2}}-\frac{a}{2 A^{2}} s^{2} \frac{A^{2} \tilde{u}}{A^{2}}\right) d s d \tau= \\
& =\int_{r}^{r+h} \frac{1}{\tau^{2}-K \tau+Q^{2}} \int_{\frac{1}{\alpha}}^{\frac{1}{\beta}}\left(\frac{s^{4}}{s^{2}-K s+Q^{2}} \frac{v^{\prime \prime}(t)}{v(t)} \frac{1}{A^{2}}-s^{2} \frac{a}{2 A^{4}}\right) A^{2} \tilde{u} d s d \tau .
\end{aligned}
$$

From i4) we have that (for $s \in\left[\frac{1}{\alpha}, \frac{1}{\beta}\right]$ )

$$
\begin{aligned}
& \frac{s^{4}}{s^{2}-K s+Q^{2}} \frac{v^{\prime \prime}(t)}{v(t)} \frac{1}{A^{2}}-\frac{a}{2 A^{2}} \frac{s^{2}}{A^{2}} \geq \\
\geq & \frac{1}{\alpha^{2}} \frac{1}{1-\alpha K+\alpha^{2} Q^{2}} \frac{a}{2 A^{6}}-\frac{1}{\beta^{2}} \frac{a}{2 A^{4}}>0 .
\end{aligned}
$$

On the other hand we have $\tilde{u} \geq \frac{1}{A^{2}}$ for every $t \in[0,1]$ and every $r \in\left[\frac{1}{\alpha}, \frac{1}{\beta}\right]$, therefore $A^{2} \tilde{u} \geq 1$ and $A^{2} \tilde{u} \leq A^{2 p} \tilde{u}^{p}$. Consequently

$$
I_{21} \leq \int_{r}^{r+h} \frac{1}{\tau^{2}-K \tau+Q^{2}} \int_{\frac{1}{\alpha}}^{\frac{1}{\beta}}\left(\frac{s^{4}}{s^{2}-K s+Q^{2}} \frac{v^{\prime \prime}(t)}{v(t)} \frac{1}{A^{2}}-a s^{2} \frac{1}{2 A^{4}}\right) A^{2 p} \tilde{u}^{p} d s d \tau .
$$

From i5) we have that

From here we get

$$
\frac{1}{1-K+Q^{2}} \leq A \leq A^{2}
$$

$$
\begin{aligned}
I_{21} \leq & \int_{r}^{r+h} \frac{1}{1-K+Q^{2}} \int_{\frac{1}{\alpha}}^{\frac{1}{\beta}}\left[\frac{v^{\prime \prime}(t)}{v(t)}-\frac{a}{2 A^{4}}\right] A^{2 p} \tilde{u}^{p} d s d \tau \leq \\
& \leq \frac{1}{1-K+Q^{2}} \frac{v^{\prime \prime}(t)-\frac{a}{2 A^{4}} v}{v} A^{2 p} \int_{0}^{1} \tilde{u}^{p} d s h= \\
& =\frac{1}{1-K+Q^{2}} \frac{v^{\prime \prime}(t)-\frac{a}{2 A^{4}} v}{v} A^{2 p}\|\tilde{u}\|_{L^{p}}^{p} h \leq \\
& \leq \frac{1}{\left(1-K+Q^{2}\right)^{2}} \frac{v^{\prime \prime}(t)-\frac{a}{2 A^{4}} v}{v} A^{2 p}\|\tilde{u}\|_{L^{p}}^{p} h .
\end{aligned}
$$

After we use the lemma 1 we get

$$
I_{21} \leq C^{p} \frac{1}{\left(1-K+Q^{2}\right)^{2}} \frac{v^{\prime \prime}(t)-\frac{a}{2 A^{4}} v}{v} A^{2 p}\|\tilde{u}\|_{\dot{B}_{p, q}^{\gamma}}^{p} h .
$$

For $I_{22}$ we have

$$
I_{22} \leq C \frac{1}{\left(1-K+Q^{2}\right)}\left(\frac{1}{1-K+Q^{2}} \frac{2 a}{A^{2}}+\frac{b}{A B}\right)\|\tilde{u}\|_{\dot{B}_{p, q}^{\gamma}} h .
$$


Consequently

$$
\begin{gathered}
I_{2} \leq\left[C \frac{1}{\left(1-K+Q^{2}\right)}\left(\frac{1}{1-K+Q^{2}} \frac{2 a}{A^{2}}+\frac{b}{A B}\right)\|\tilde{u}\|_{\dot{B}_{p, q}^{\gamma},} h+\right. \\
\left.+C^{p} \frac{1}{\left(1-K+Q^{2}\right)^{2}} \frac{v^{\prime \prime}(t)-\frac{a}{2 A^{4}} v}{v} A^{2 p}\|\tilde{u}\|_{\dot{B}_{p, q}^{\gamma}}^{p} h\right]^{p}, \\
\left\|\Delta_{h} R(\tilde{u})\right\|_{L^{p}}^{q} \leq\left[\left[C \frac{1}{\left(1-K+Q^{2}\right)}\left(\frac{1}{1-K+Q^{2}} \frac{2 a}{A^{2}}+\frac{b}{A B}\right)\|\tilde{u}\|_{\dot{B}_{p, q}^{\gamma}} h+\right.\right. \\
\left.+C^{p} \frac{1}{\left(1-K+Q^{2}\right)^{2}} \frac{v^{\prime \prime}(t)-\frac{a}{2 A^{4}} v}{v} A^{2 p}\|\tilde{u}\|_{\dot{B}_{p, q}^{\gamma}}^{p} h\right]^{p}+ \\
\left.C^{p} \frac{1}{\left(1-K+Q^{2}\right)^{p}}\left(\frac{1}{1-K+Q^{2}} \frac{2 a}{A^{2}}+\frac{b}{A B}\right)^{p}\|\tilde{u}\|_{\dot{B}_{p, q}^{\gamma}}^{p} h^{p}\right]^{\frac{q}{p}} .
\end{gathered}
$$

Then

Let

$$
\begin{aligned}
\|\tilde{u}\|_{\dot{B}_{p, q}^{\gamma}} & \leq C \frac{2^{2-\gamma}}{\left(1-K+Q^{2}\right)(q(1-\gamma))^{\frac{1}{q}}}\left(\frac{1}{1-K+Q^{2}} \frac{2 a}{A^{2}}+\frac{b}{A B}\right)\|\tilde{u}\|_{\dot{B}_{p, q}^{\gamma}}+ \\
& +C^{p} \frac{2^{1-\gamma}}{\left(1-K+Q^{2}\right)^{2}(q(1-\gamma))^{\frac{1}{q}}} \frac{v^{\prime \prime}(t)-\frac{a}{2 A^{4}} v}{v} A^{2 p}\|\tilde{u}\|_{\dot{B}_{p, q}^{\gamma}}^{p} .
\end{aligned}
$$

$$
\begin{gathered}
C_{1}=C \frac{2^{2-\gamma}}{(q(1-\gamma))^{\frac{1}{q}}\left(1-K+Q^{2}\right)}\left(\frac{1}{1-K+Q^{2}} \frac{2 a}{A^{2}}+\frac{b}{A B}\right), \\
D=v\left(1-K+Q^{2}\right)^{2}(q(1-\gamma))^{\frac{1}{q}} \frac{1}{C^{p} 2^{1-\gamma} A^{2 p}} .
\end{gathered}
$$

From i3) we have that $C_{1}<1$. Then

$$
\|\tilde{u}\|_{\dot{B}_{p, q}^{\gamma}} \leq C_{1}\|\tilde{u}\|_{\dot{B}_{p, q}^{\gamma}}+\frac{v^{\prime \prime}(t)-\frac{a}{2 A^{4}} v}{D}\|\tilde{u}\|_{\dot{B}_{p, q}^{\gamma}}^{p},
$$

from here

$$
\frac{\left(1-C_{1}\right) D}{v^{\prime \prime}(t)-\frac{a}{2 A^{4}} v} \leq\|\tilde{u}\|_{\dot{B}_{p, q}^{\gamma}}^{p-1} .
$$

Since

we have

$$
\lim _{t \longrightarrow 0}\left[v^{\prime \prime}(t)-\frac{a}{2 A^{4}} v\right]=+0,
$$

$$
\lim _{t \longrightarrow 0}\|\tilde{u}\|_{\dot{B}_{p, q}^{\gamma}}=\infty
$$

\section{Proof of Theorem 2.}

4.1. Local existence of nontrivial solutions for nonhomogenious Cauchy problem (1), (2). Let $v(t)$ is fixed function which satisfies the conditions (H1), (H2) and (H4).

Let us consider the equation

$$
u(t, r)=\left\{\begin{array}{l}
\int_{r}^{r_{1}} \frac{1}{\tau^{2}-K \tau+Q^{2}} \int_{\tau}^{r_{1}}\left(\frac{s^{4}}{s^{2}-K s+Q^{2}} \frac{v^{\prime \prime}(t)}{v(t)} u(t, s)-s^{2} f(u(t, s))-s^{2} g(s)\right) d s d \tau, \\
0 \leq r \leq r_{1}, \\
0 \quad \text { for } \quad r \geq r_{1},
\end{array}\right.
$$

$t \in[0,1]$, where $u(t, r)=v(t) \omega(r)$. 
Theorem 4.1. Let $v(t)$ is fixed function which satisfies the hypothesis (H1), (H2), (H4). Let also $p>1, q \in[1, \infty)$ and $\gamma \in(0,1)$ are fixed and the positive constants $A, a, b, B, Q, K, \alpha>\beta>1$ satisfy the conditions i2), i3), i5)-i7) and $f \in \mathcal{C}^{1}\left(\mathcal{R}^{1}\right), f(0)=0, a|u| \leq f^{\prime}(u) \leq b|u|, g \in \mathcal{C}\left(\mathcal{R}^{+}\right), g(r) \geq 0$ for $\forall r \in$ $\mathcal{R}^{+}, g(r)=0$ for $r \geq r_{1}$. Then the equation $\left(\star^{\prime}\right)$ has unique nontrivial solution $u(t, r)=v(t) \omega(r)$ for which $w \in \mathcal{C}^{2}\left[0, r_{1}\right], u(t, r)=u_{r}(t, r)=u_{r r}(t, r)=0$ for $r \geq r_{1}, u(t, r) \in \mathcal{C}\left((0,1] \dot{B}_{p, q}^{\gamma}\left[0, r_{1}\right]\right)$, for $r \in\left[\frac{1}{\alpha}, \frac{1}{\beta}\right]$ and $t \in[0,1] u(t, r) \geq \frac{1}{A^{2}}$, for $r \in\left[0, r_{1}\right]$ and $t \in[0,1]|u(t, r)| \leq \frac{2}{A B}$.

Proof. Let $N_{1}$ is the set

$$
\begin{gathered}
N_{1}=\left\{u(t, r) \in \mathcal{C}\left([0,1] \times\left[0, r_{1}\right]\right) ; u(t, r)=u_{r}(t, r)=u_{r r}(t, r)=0\right. \\
\qquad \text { for } r \geq r_{1} \quad \text { and } t \in[0,1], \\
u(t, r) \in \mathcal{C}\left((0,1] \dot{B}_{p, q}^{\gamma}\left[0, r_{1}\right]\right) ; \text { for } \quad r \in\left[\frac{1}{\alpha}, \frac{1}{\beta}\right] \quad \text { and } \quad t \in[0,1] \quad u(t, r) \geq \frac{1}{A^{2}} \\
\text { for } \left.r \in\left[0, r_{1}\right] \quad \text { and } \quad t \in[0,1] \quad|u(t, r)| \leq \frac{2}{A B}\right\} .
\end{gathered}
$$

Let also $t \in[0,1]$ is fixed.

We define the operator $R_{1}$ as follow

$R_{1}(u)=\int_{r}^{r_{1}} \frac{1}{\tau^{2}-K \tau+Q^{2}} \int_{\tau}^{r_{1}}\left(\frac{s^{4}}{s^{2}-K s+Q^{2}} \frac{v^{\prime \prime}(t)}{v(t)} u(t, s)-s^{2} f(u)-s^{2} g(s)\right) d s d \tau$, where $0 \leq r \leq r_{1}$, and $t \in[0,1]$. First we will show that $R_{1}: N_{1} \longrightarrow N_{1}$. We have

1) $R_{1}(u) \in \mathcal{C}^{2}\left(\left[0, r_{1}\right]\right),\left.R_{1}(u)\right|_{r=r_{1}}=0$,

$$
\begin{gathered}
\frac{\partial}{\partial r} R_{1}(u)=\frac{1}{r^{2}-K r+Q^{2}} \int_{r_{1}}^{r}\left[\frac{s^{4}}{s^{2}-K s+Q^{2}} \frac{v^{\prime \prime}(t)}{v(t)} u\right. \\
\left.-s^{2} f(u)-s^{2} g(s)\right] d s,\left.\frac{\partial}{\partial r} R_{1}(u)\right|_{r=r_{1}}=0, \\
\frac{\partial^{2}}{\partial r^{2}} R_{1}(u)=\frac{K-2 r}{\left(r^{2}-K r+Q^{2}\right)^{2}} \int_{r_{1}}^{r}\left[\frac{s^{4}}{s^{2}-K s+Q^{2}} \frac{v^{\prime \prime}(t)}{v(t)} u-s^{2} f(u)-s^{2} g(s)\right] d s+ \\
+\frac{r^{4}}{\left(r^{2}-K r+Q^{2}\right)^{2}} \frac{v^{\prime \prime}(t)}{v(t)} u(t, r)-\frac{r^{2}}{r^{2}-K r+Q^{2}}(f(u)+g(r)) .
\end{gathered}
$$

Since $u\left(t, r_{1}\right)=0, f\left(u\left(t, r_{1}\right)\right)=f(0)=0$ and $g\left(r_{1}\right)=0$ we get that

$$
\left.\frac{\partial^{2}}{\partial r^{2}} R_{1}(u)\right|_{r=r_{1}}=0 \text {. }
$$

2) For $r \in\left[0, r_{1}\right]$ and $t \in[0,1]$ we have $|u(t, r)| \leq \frac{2}{A B}$. Then

$$
\begin{gathered}
\left|R_{1}(u)\right|=\left|\int_{r_{1}}^{r} \frac{1}{\tau^{2}-K \tau+Q^{2}} \int_{r_{1}}^{\tau}\left(\frac{s^{4}}{s^{2}-K s+Q^{2}} \frac{v^{\prime \prime}(t)}{v(t)} u-s^{2} f(u)-s^{2} g(s)\right) d s d \tau\right| \leq \\
\leq \int_{r_{1}}^{r} \frac{1}{\tau^{2}-K \tau+Q^{2}} \int_{r_{1}}^{\tau}\left(\frac{s^{4}}{s^{2}-K s+Q^{2}} \frac{v^{\prime \prime}(t)}{v(t)}|u|+s^{2}|f(u)|+s^{2}|g(s)|\right) d s d \tau \leq
\end{gathered}
$$

(since $\left|f^{\prime}(u)\right| \leq b|u|$ and $f(0)=0$ we have $|f(u)| \leq \frac{b}{2}|u|^{2}$ )

$$
\begin{gathered}
\leq \int_{r_{1}}^{r} \frac{1}{\tau^{2}-K \tau+Q^{2}} \int_{r_{1}}^{\tau}\left(\frac{s^{4}}{s^{2}-K s+Q^{2}} \frac{v^{\prime \prime}(t)}{v(t)}|u|+\frac{b}{2} s^{2}|u|^{2}+s^{2}|g(s)|\right) d s d \tau= \\
\int_{r_{1}}^{r} \frac{1}{\tau^{2}-K \tau+Q^{2}} \int_{r_{1}}^{\tau}\left(\left(\frac{s^{4}}{s^{2}-K s+Q^{2}} \frac{v^{\prime \prime}(t)}{v(t)}+\frac{b}{2} s^{2}|u|\right)|u|+s^{2}|g(s)|\right) d s d \tau \leq
\end{gathered}
$$




$$
\begin{gathered}
\leq \int_{r_{1}}^{r} \frac{1}{\tau^{2}-K \tau+Q^{2}} \int_{r_{1}}^{\tau}\left(\frac{2}{A B}\left(\frac{s^{4}}{s^{2}-K s+Q^{2}} \frac{v^{\prime \prime}(t)}{v(t)}+\frac{b}{2} \frac{2}{A B} s^{2}\right)\right. \\
\left.+s^{2} \max _{s \in\left[0, r_{1}\right]}|g(s)|\right) d s d \tau \leq
\end{gathered}
$$

(here we use that $\frac{r^{2}}{r^{2}-K r+Q^{2}} \leq \frac{1}{1-K+Q^{2}}, \frac{1}{r^{2}-K r+Q^{2}} \leq \frac{1}{1-K+Q^{2}}$ for $r \in\left[0, r_{1}\right]$ )

$$
\begin{aligned}
\leq & \frac{1}{1-K+Q^{2}}\left(\frac{2}{A B}\left(\frac{1}{1-K+Q^{2}} \max _{t \in[0,1]} \frac{v^{\prime \prime}(t)}{v(t)}+\frac{b}{A B}\right)+s^{2} \max _{s \in\left[0, r_{1}\right]}|g(s)|\right) \leq \\
& \leq \frac{1}{1-K+Q^{2}}\left(\frac{2}{A B}\left(\frac{1}{1-K+Q^{2}} \frac{2 a}{A^{2}}+\frac{b}{A B}\right)+r_{1}^{2} \max _{s \in\left[0, r_{1}\right]}|g(s)|\right) \leq \frac{2}{A B}
\end{aligned}
$$

(in the last inequality we use i6)). Consequently

$$
\left|R_{1}(u)\right| \leq \frac{2}{A B} \quad \text { for } \quad r \in\left[0, r_{1}\right], \quad t \in[0,1] .
$$

3) For $r \in\left[\frac{1}{\alpha}, \frac{1}{\beta}\right]$,

(5) $\frac{\partial}{\partial r} R_{1}(u)=\frac{1}{r^{2}-K r+Q^{2}} \int_{r}^{r_{1}}\left(s^{2} f(u)+s^{2} g(s)-\frac{v^{\prime \prime}(t)}{v(t)} \frac{s^{4}}{s^{2}-K s+Q^{2}} u\right) d s \geq$ (as in point 3) of the proof of theorem 2.1)

$$
\geq \frac{1}{r^{2}-K r+Q^{2}} \int_{r}^{r_{1}}\left(s^{2} f(u)-\frac{v^{\prime \prime}(t)}{v(t)} \frac{s^{4}}{s^{2}-K s+Q^{2}} u\right) d s \geq 0 .
$$

From (5) we conclude that $R_{1}(u)$ is increase function for $r \in\left[\frac{1}{\alpha}, \frac{1}{\beta}\right]$. Consequently, for $r \in\left[\frac{1}{\alpha}, \frac{1}{\beta}\right]$,

$$
\begin{aligned}
& R_{1}(u) \geq \int_{\frac{1}{\alpha}}^{r_{1}} \frac{1}{\tau^{2}-K \tau+Q^{2}} \int_{\tau}^{r_{1}}\left(\frac{v^{\prime \prime}(t)}{v(t)} \frac{s^{4}}{s^{2}-K s+Q^{2}} u-s^{2} f(u)-s^{2} g(s)\right) d s d \tau \geq \\
& \geq \int_{\frac{1}{\alpha}}^{\frac{1}{\beta}} \frac{\alpha^{2}}{1-\alpha K+\alpha^{2} Q^{2}} \int_{\frac{1}{\alpha}}^{\frac{1}{\beta}} \frac{1}{\alpha^{2}\left(1-K \alpha+\alpha^{2} Q^{2}\right)} \frac{a}{4 A^{6}} d s d \tau- \\
&-r_{1}^{2} \frac{2 b}{\left(1-K+Q^{2}\right) A^{2} B^{2}}-r_{1}^{2} \max _{r \in\left[0, r_{1}\right]} g(r) \frac{1}{1-K+Q^{2}} \geq \\
& \geq\left(\frac{1}{\beta}-\frac{1}{\alpha}\right)^{2} \frac{1}{\left(1-\alpha K+\alpha^{2} Q^{2}\right)^{2}} \frac{a}{4 A^{6}}- \\
&-r_{1}^{2} \frac{2 b}{\left(1-K+Q^{2}\right) A^{2} B^{2}}-r_{1}^{2} \frac{1}{1-K+Q^{2}} \max _{r \in\left[0, r_{1}\right]} g(r) \geq \frac{1}{A^{2}} .
\end{aligned}
$$

(see fourth inequality of i2)) Consequently for $r \in\left[\frac{1}{\alpha}, \frac{1}{\beta}\right]$ and $t \in[0,1]$ we have

$$
R_{1}(u) \geq \frac{1}{A^{2}}
$$

4)

$$
\begin{gathered}
\left\|\Delta_{h} R_{1}(u)\right\|_{L^{p}}^{q}= \\
=\left(\int _ { 0 } ^ { r _ { 1 } } \left(\mid \int_{r}^{r+h} \frac{1}{\tau^{2}-K \tau+Q^{2}} \int_{\tau}^{r_{1}}\left(\frac{s^{4}}{s^{2}-K s+Q^{2}} \frac{v^{\prime \prime}(t)}{v(t)} u(t, s)\right.\right.\right. \\
\left.\left.\left.-s^{2} f(u)-s^{2} g(s)\right) d s d \tau \mid\right)^{p} d r\right)^{\frac{q}{p}} \leq
\end{gathered}
$$




$$
\begin{gathered}
\leq\left(\int _ { 0 } ^ { r _ { 1 } } \left(\int _ { r } ^ { r + h } \frac { 1 } { \tau ^ { 2 } - K \tau + Q ^ { 2 } } \int _ { \tau } ^ { r _ { 1 } } \left(\frac{s^{4}}{s^{2}-K s+Q^{2}} \frac{v^{\prime \prime}(t)}{v(t)}|u|+\right.\right.\right. \\
\left.\left.\left.\frac{b}{2}|u|^{2} s^{2}+s^{2} g(s)\right) d s d \tau\right)^{p} d r\right)^{\frac{q}{p}} \leq
\end{gathered}
$$

(here we use that for $s \in\left[0, r_{1}\right]$ we have $\frac{s^{4}}{s^{2}-K s+Q^{2}} \leq \frac{1}{1-K+Q^{2}}$, also for $r \in\left[0, r_{1}\right]$ and $t \in[0,1]$ we have $\left.|u(t, r)| \leq \frac{2}{A B}\right)$

$$
\begin{gathered}
\leq\left(\int _ { 0 } ^ { r _ { 1 } } \left(\int _ { r } ^ { r + h } \frac { 1 } { 1 - K + Q ^ { 2 } } \int _ { \tau } ^ { r _ { 1 } } \left(\frac{1}{1-K+Q^{2}} \max _{t \in[0,1]} \frac{v^{\prime \prime}(t)}{v(t)}|u|+\right.\right.\right. \\
\left.\left.\left.+\frac{b}{A B}|u|+\max _{s \in\left[0, r_{1}\right]}|g(s)|\right) d s d \tau\right)^{p} d r\right)^{\frac{q}{p}} \leq
\end{gathered}
$$

(here we use (H4))

$$
\begin{gathered}
\leq\left(\int _ { 0 } ^ { r _ { 1 } } \left(\int _ { r } ^ { r + h } \frac { 1 } { 1 - K + Q ^ { 2 } } \int _ { \tau } ^ { r _ { 1 } } \left(\frac{1}{1-K+Q^{2}} \frac{2 a}{A^{2}}|u|+\right.\right.\right. \\
\left.\left.\left.\frac{b}{A B}|u|+\max _{s \in\left[0, r_{1}\right]}|g(s)|\right) d s d \tau\right)^{p} d r\right)^{\frac{q}{p}} \leq \\
\leq\left(\int _ { 0 } ^ { r _ { 1 } } \left(\int_{r}^{r+h} \frac{1}{\left(1-K+Q^{2}\right)^{2}} \frac{2 a}{A^{2}} \int_{0}^{r_{1}}|u| d s+\frac{1}{1-K+Q^{2}} \frac{b}{A B} \int_{0}^{r_{1}}|u| d s+\right.\right. \\
\left.\left.\left.+\frac{1}{1-K+Q^{2}} \max _{s \in\left[0, r_{1}\right]}|g(s)|\right) d \tau\right)^{p} d r\right)^{\frac{q}{p}} \leq \\
\leq h^{q}\left(\frac{1}{\left(1-K+Q^{2}\right)^{2}} \frac{2 a}{A^{2}}\|u\|_{L^{p}}+\right. \\
\left.\frac{1}{1-K+Q^{2}} \frac{b}{A B}\left|u \|_{L^{p}}+\frac{1}{1-K+Q^{2}} \max _{s \in\left[0, r_{1}\right]}\right| g(s) \mid\right)^{q},
\end{gathered}
$$

i.e.

$$
\begin{aligned}
\left\|\Delta_{h} R_{1}(u)\right\|_{L^{p}}^{q} \leq h^{q} & \left(\frac{1}{\left(1-K+Q^{2}\right)^{2}} \frac{2 a}{A^{2}}\|u\|_{L^{p}}+\frac{1}{1-K+Q^{2}} \frac{b}{A B}\|u\|_{L^{p}}+\right. \\
& \left.+\frac{1}{1-K+Q^{2}} \max _{s \in\left[0, r_{1}\right]}|g(s)|\right)^{q} .
\end{aligned}
$$

Consequently

$$
\begin{aligned}
& \left\|R_{1}(u)\right\|_{\dot{B}_{p}^{\gamma}, q\left(0, r_{1}\right)}^{q}=\int_{0}^{2} h^{-1-q \gamma}\left\|\Delta_{h} R_{1}(u)\right\|_{L^{p}}^{q} d h \leq \\
& \leq\left(\frac{1}{\left(1-K+Q^{2}\right)^{2}} \frac{2 a}{A^{2}}\|u\|_{L^{p}}+\frac{b}{A B\left(1-K+Q^{2}\right)}\|u\|_{L^{p}}+\right. \\
& \left.+\frac{1}{1-K+Q^{2}} \max _{s \in\left[0, r_{1}\right]}|g(s)|\right)^{q} \int_{0}^{2} h^{-1+q(1-\gamma)} d h= \\
& =\left(\frac{1}{\left(1-K+Q^{2}\right)^{2}} \frac{2 a}{A^{2}}\|u\|_{L^{p}}+\frac{b}{A B\left(1-K+Q^{2}\right)}\|u\|_{L^{p}}\right. \\
& \left.+\frac{1}{1-K+Q^{2}} \max _{s \in\left[0, r_{1}\right]}|g(s)|\right)^{q} \frac{2^{q(1-\gamma)}}{q(1-\gamma)} .
\end{aligned}
$$

Therefore

$$
\left\|R_{1}(u)\right\|_{\dot{B}_{p, q}^{\gamma}\left[0, r_{1}\right]} \leq\left(\frac{1}{\left(1-K+Q^{2}\right)^{2}} \frac{2 a}{A^{2}}\|u\|_{L^{p}}+\frac{b}{A B\left(1-K+Q^{2}\right)}\|u\|_{L^{p}}+\right.
$$




$$
\left.+\frac{1}{1-K+Q^{2}} \max _{s \in\left[0, r_{1}\right]}|g(s)|\right) \frac{2^{(1-\gamma)}}{(q(1-\gamma))^{\frac{1}{q}}} .
$$

From lemma 1 we get

$$
\begin{gathered}
\left\|R_{1}(u)\right\|_{\dot{B}_{p, q}^{\gamma}\left[0, r_{1}\right]} \leq\left(C \frac{1}{\left(1-K+Q^{2}\right)^{2}} \frac{2 a}{A^{2}}\|u\|_{\dot{B}_{p, q}^{\gamma}\left[0, r_{1}\right]}\right. \\
+C \frac{b}{A B\left(1-K+Q^{2}\right)}\|u\|_{\dot{B}_{p, q}^{\gamma}\left[0, r_{1}\right]}+ \\
\left.+\frac{1}{1-K+Q^{2}} \max _{s \in\left[0, r_{1}\right]}|g(s)|\right) \frac{2^{1-\gamma}}{(q(1-\gamma))^{\frac{1}{q}}} .
\end{gathered}
$$

From the last inequality, if $u \in \dot{B}_{p, q}^{\gamma}\left[0, r_{1}\right]$ for $t \in[0,1]$ we get $R_{1}(u) \in \dot{B}_{p, q}^{\gamma}\left[0, r_{1}\right]$ for $t \in[0,1]$. Also $R_{1}(u) \in \mathcal{C}\left((0,1] \dot{B}_{p, q}^{\gamma}\left[0, r_{1}\right]\right)$.

From 1), 2), 3), 4) we get $R_{1}: N_{1} \longrightarrow N_{1}$.

Let $u, u_{1} \in N_{1}$. Then

$$
R_{1}(u)-R_{1}\left(u_{1}\right)=R(u)-R\left(u_{1}\right) .
$$

From here and from the proof of theorem 2.1 we get

$$
\begin{gathered}
\left\|R_{1}(u)-R_{1}\left(u_{1}\right)\right\|_{\dot{B}_{p, q}^{\gamma}\left[0, r_{1}\right]} \leq \\
\leq C\left(\frac{1}{\left(1-K+Q^{2}\right)^{2}} \frac{2 a}{A^{2}}\left\|u-u_{1}\right\|_{\dot{B}_{p, q}^{\gamma}\left[0, r_{1}\right]}+\right. \\
\left.\frac{2 b}{A B\left(1-K+Q^{2}\right)}\left\|u-u_{1}\right\|_{\dot{B}_{p, q}^{\gamma}\left[0, r_{1}\right]}\right) \frac{2^{1-\gamma}}{(q(1-\gamma))^{\frac{1}{q}}} .
\end{gathered}
$$

From i3) we have

$$
C\left(\frac{1}{\left(1-K+Q^{2}\right)^{2}} \frac{2 a}{A^{2}}+\frac{2 b}{A B\left(1-K+Q^{2}\right)}\right) \frac{2^{1-\gamma}}{(q(1-\gamma))^{\frac{1}{q}}}<1 .
$$

Therefore

$$
\left\|R_{1}(u)-R_{1}\left(u_{1}\right)\right\|_{\dot{B}_{p, q}^{\gamma}\left[0, r_{1}\right]}<\left\|u-u_{1}\right\|_{\dot{B}_{p, q}^{\gamma}\left[0, r_{1}\right]} .
$$

Consequently the operator $R_{1}: N_{1} \longrightarrow N_{1}$ is contractive operator. Since $N_{1}$ is closed subset of $\mathcal{C}\left((0,1] \dot{B}_{p, q}^{\gamma}\left[0, r_{1}\right]\right)$ (see appendix of this paper) we conclude that $\left(\star^{\prime}\right)$ has unique nontrivial solution in the set $N$. $\bullet$

Let $\bar{u}$ is the solution from the theorem 4. 1. , i.e $\overline{\bar{u}}$ is the solution to the equation $\left(\star^{\prime}\right)$. From proposition 2.3 we have that $\bar{u}$ satisfies the equation (1). Then $\bar{u}$ is solution to the Cauchy problem (1), (2) with initial data

$$
\begin{aligned}
& \overline{\bar{u}}_{0}=\left\{\begin{array}{l}
\int_{r}^{r_{1}} \frac{1}{\tau^{2}-K \tau+Q^{2}} \int_{\tau}^{r_{1}}\left(\frac{s^{4}}{s^{2}-K s+Q^{2}} v^{\prime \prime}(1) \omega(s)-s^{2} f(v(1) \omega(s))-s^{2} g(s)\right) d s d \tau \\
0 \quad \text { for } r \geq r_{1},
\end{array}\right. \\
& \overline{\bar{u}}_{1}=\left\{\begin{array}{l}
\int_{r}^{r_{1}} \frac{1}{\tau^{2}-K \tau+Q^{2}} \int_{\tau}^{r_{1}}\left(\frac{s^{4}}{s^{2}-K s+Q^{2}} v^{\prime \prime \prime}(1) \omega(s)-s^{2} f^{\prime}(u) v^{\prime}(1) \omega(s)\right) d s d \tau \equiv 0 \\
0 \quad \text { for } r \geq r_{1},
\end{array}\right. \\
& \overline{\bar{u}}_{0} \in \dot{B}_{p, q}^{\gamma}\left(\mathcal{R}^{+}\right), \overline{\bar{u}}_{1} \in \dot{B}_{p, q}^{\gamma-1}\left(\mathcal{R}^{+}\right), \overline{\bar{u}} \in \mathcal{C}\left((0,1] \dot{B}_{p, q}^{\gamma}\left[0, r_{1}\right]\right) .
\end{aligned}
$$


4.2. Blow up of the solutions to the nonhomogeneous Cauchy problem (1), (2). Let $v(t)$ is same function as in Theorem 4.1.

Theorem 4.2. Let $p>1, q \geq 1$ and $\gamma \in(0,1)$ are fixed and the positive constants $a, b, A, B, Q, K, 1<\beta<\alpha$ satisfy the conditions i2)-i7). Let $f \in$ $\mathcal{C}^{1}\left(\mathcal{R}^{1}\right), f(0)=0, a|u| \leq f^{\prime}(u) \leq b|u|, g \in \mathcal{C}\left(\mathcal{R}^{+}\right), g(r) \geq 0$ for $r \geq 0, g(r)=0$ for $r \geq r_{1}$. Then for the solution $\overline{\bar{u}}$ to the Cauchy problem (1), (2) we have

$$
\lim _{t \longrightarrow 0}\|\overline{\bar{u}}\|_{\dot{B}_{p, q}^{\gamma}\left[0, r_{1}\right]}=\infty .
$$

Proof. We suppose that $t \in(0,1]$. Then we have

$$
\begin{gathered}
\left\|\Delta_{h} \bar{u}\right\|_{L^{p}}^{q}= \\
=\left(\int_{0}^{r_{1}} \mid \int_{r}^{r+h} \frac{1}{\tau^{2}-K \tau+Q^{2}} \int_{\tau}^{r_{1}}\left[\frac{s^{4}}{s^{2}-K s+Q^{2}} \frac{v^{\prime \prime}(t)}{v(t)} \bar{u}\right.\right. \\
\left.\left.-s^{2} f(\bar{u})-s^{2} g(s)\right]\left.d s d \tau\right|^{p} d r\right)^{\frac{q}{p}}= \\
=\left(\int_{0}^{\frac{1}{\alpha}} \mid \int_{r}^{r+h} \frac{1}{\tau^{2}-K \tau+Q^{2}} \int_{\tau}^{r_{1}}\left[\frac{s^{4}}{s^{2}-K s+Q^{2}} \frac{v^{\prime \prime}(t)}{v(t)} \bar{u}\right.\right. \\
+\int_{\frac{1}{\alpha}}^{r_{1}} \mid \int_{r}^{r+h} \frac{1}{\tau^{2}-K \tau+Q^{2}} \int_{\tau}^{r_{1}}\left[\frac{s^{4}}{s^{2}-K s+Q^{2}} \frac{v^{\prime \prime}(t)}{v(t)} \bar{u}\right. \\
\left.\left.-s^{2} f(\bar{u})-s^{2} g(s)\right]\left.d s d \tau\right|^{p} d r\right)^{\frac{q}{p}} .
\end{gathered}
$$

Let

$$
\begin{gathered}
I_{1}=\int_{0}^{\frac{1}{\alpha}} \mid \int_{r}^{r+h} \frac{1}{\tau^{2}-K \tau+Q^{2}} \int_{\tau}^{r_{1}}\left[\frac{s^{4}}{s^{2}-K s+Q^{2}} \frac{v^{\prime \prime}(t)}{v(t)} \bar{u}\right. \\
\left.-s^{2} f(\bar{u})-s^{2} g(s)\right]\left.d s d \tau\right|^{p} d r, \\
I_{2}=\int_{\frac{1}{\alpha}}^{r_{1}} \mid \int_{r}^{r+h} \frac{1}{\tau^{2}-K \tau+Q^{2}} \int_{\tau}^{r_{1}}\left[\frac{s^{4}}{s^{2}-K s+Q^{2}} \frac{v^{\prime \prime}(t)}{v(t)} \bar{u}\right. \\
\left.-s^{2} f(\bar{u})-s^{2} g(s)\right]\left.d s d \tau\right|^{p} d r .
\end{gathered}
$$

Then

$$
\left\|\Delta_{h} \bar{u}\right\|_{L^{p}\left[0, r_{1}\right]}^{q} \leq\left(I_{1}+I_{2}\right)^{\frac{q}{p}}
$$

For $I_{1}$ we have the following estimate

$$
\begin{gathered}
I_{1} \leq \int_{0}^{\frac{1}{\alpha}}\left(\int _ { r } ^ { r + h } \frac { 1 } { \tau ^ { 2 } - K \tau + Q ^ { 2 } } \int _ { \tau } ^ { r _ { 1 } } \left[\frac{s^{4}}{s^{2}-K s+Q^{2}} \frac{v^{\prime \prime}(t)}{v(t)}|\bar{u}|\right.\right. \\
\left.\left.+s^{2} f(\bar{u})+s^{2} g(s)\right] d s d \tau\right)^{p} d r \leq
\end{gathered}
$$

(here we use that $r_{1}<1$ )

$$
\begin{gathered}
\leq \int_{0}^{\frac{1}{\alpha}}\left(\int _ { r } ^ { r + h } \frac { 1 } { \tau ^ { 2 } - K \tau + Q ^ { 2 } } \int _ { \tau } ^ { r _ { 1 } } \left[\frac{s^{4}}{s^{2}-K s+Q^{2}} \frac{v^{\prime \prime}(t)}{v(t)} \bar{u}\right.\right. \\
\left.\left.+s^{2} f(\bar{u})+g(s)\right] d s d \tau\right)^{p} d r \leq
\end{gathered}
$$




$$
\begin{gathered}
\leq \int_{0}^{\frac{1}{\alpha}}\left(\int _ { r } ^ { r + h } \frac { 1 } { \tau ^ { 2 } - K \tau + Q ^ { 2 } } \int _ { \tau } ^ { r _ { 1 } } \left[\frac{s^{4}}{s^{2}-K s+Q^{2}} \frac{v^{\prime \prime}(t)}{v(t)}|\bar{u}|\right.\right. \\
\left.\left.+s^{2} f(\bar{u})+\max _{s \in\left[0, r_{1}\right]} g(s)\right] d s d \tau\right)^{p} d r .
\end{gathered}
$$

From i7) we have

$$
\max _{s \in\left[0, r_{1}\right]} g(s) \leq\left(\frac{1}{\beta}-\frac{1}{\alpha}\right)^{2} \frac{1}{A^{4}}
$$

Therefore

$$
\max _{s \in\left[0, r_{1}\right]} g(s) \leq \int_{\frac{1}{\alpha}}^{\frac{1}{\beta}} \int_{\frac{1}{\alpha}}^{\frac{1}{\beta}} \frac{1}{A^{4}} d s d \tau .
$$

Also $\bar{u} \geq \frac{1}{A^{2}}$ for $r \in\left[\frac{1}{\alpha}, \frac{1}{\beta}\right]$. Consequently

$$
\max _{s \in\left[0, r_{1}\right]} g(s) \leq \frac{1}{A^{2}} \int_{\frac{1}{\alpha}}^{\frac{1}{\beta}} \int_{\frac{1}{\alpha}}^{\frac{1}{\beta}}|\bar{u}| d s d \tau \leq \frac{1}{A^{2}} \int_{0}^{r_{1}} \int_{0}^{r_{1}}|\bar{u}| d s d \tau \leq
$$

now we use Hölder's inequality

$$
\leq \frac{1}{A^{2}}\|\bar{u}\|_{L^{p}} \leq
$$

now we use lemma 1

$$
\leq \frac{1}{A^{2}} C|| \bar{u} \|_{\dot{B}_{p, q}^{\gamma}\left[0, r_{1}\right]} .
$$

From the last inequality we get

$$
\begin{aligned}
I_{1} \leq \int_{0}^{\frac{1}{\alpha}} & \left(\int _ { r } ^ { r + h } \frac { 1 } { \tau ^ { 2 } - K \tau + Q ^ { 2 } } \int _ { \tau } ^ { r _ { 1 } } \left[\frac{s^{4}}{s^{2}-K s+Q^{2}} \frac{v^{\prime \prime}(t)}{v(t)}|\bar{u}|\right.\right. \\
& \left.\left.+s^{2} f(\bar{u})+C \frac{1}{A^{2}}\|\bar{u}\|_{\dot{B}_{p, q}^{\gamma}\left[0, r_{1}\right]}\right] d s d \tau\right)^{p} d r .
\end{aligned}
$$

From here and as in the proof of theorem 3.1 we have

$$
I_{1} \leq\left(C \frac{1}{1-K+Q^{2}}\left(\frac{1}{1-K+Q^{2}} \frac{2 a}{A^{2}}+\frac{2 b}{A B}+\frac{1}{A^{2}}\right)\|\bar{u}\|_{\dot{B}_{p, q}^{\gamma}}\right)^{p} h^{p} .
$$

For $I_{2}$ we have

$$
\begin{aligned}
I_{2}= & \int_{\frac{1}{\alpha}}^{r_{1}} \mid \int_{r}^{r+h} \frac{1}{\tau^{2}-K \tau+Q^{2}} \int_{\frac{1}{\alpha}}^{\frac{1}{\beta}}\left[\frac{s^{4}}{s^{2}-K s+Q^{2}} \frac{v^{\prime \prime}(t)}{v(t)} \bar{u}\right. \\
& +\int_{r}^{r+h} \frac{1}{\tau^{2}-K \tau+Q^{2}} \int_{\frac{1}{\beta}}^{r_{1}}\left[\frac{s^{4}}{s^{2}-K s+Q^{2}} \frac{v^{\prime \prime}(t)}{v(t)} \bar{u}\right. \\
\leq & \int_{\frac{1}{\alpha}}^{r_{1}}\left(\int _ { r } ^ { r + h } \frac { 1 } { \tau ^ { 2 } - K \tau + Q ^ { 2 } } \int _ { \frac { 1 } { \alpha } } ^ { \frac { 1 } { \beta } } \left[\frac{s^{4}}{s^{2}-K s+Q^{2}} \frac{v^{\prime \prime}(t)}{v(t)} \bar{u}\right.\right. \\
& +\mid \int_{r}^{r+h} \frac{1}{\tau^{2}-K \tau+s^{2}} \int_{\frac{1}{\beta}}^{r_{1}}\left[\frac{s^{4}}{s^{2}-K s+Q^{2}} \frac{v^{\prime \prime}(t)}{v(t)} \bar{u}\right.
\end{aligned}
$$


Let

$$
\left.\left.-s^{2} f(\bar{u})-s^{2} g(s)\right] d s d \tau \mid\right)^{p} d r
$$

Then

$$
\begin{gathered}
I_{21}=\int_{r}^{r+h} \frac{1}{\tau^{2}-K \tau+Q^{2}} \int_{\frac{1}{\alpha}}^{\frac{1}{\beta}}\left[\frac{s^{4}}{s^{2}-K s+Q^{2}} \frac{v^{\prime \prime}(t)}{v(t)} \bar{u}\right. \\
\left.\quad-s^{2} f(\bar{u})-s^{2} g(s)\right] d s d \tau, \\
I_{22}=\mid \int_{r}^{r+h} \frac{1}{\tau^{2}-K \tau+Q^{2}} \int_{\frac{1}{\beta}}^{r_{1}}\left[\frac{s^{4}}{s^{2}-K s+Q^{2}} \frac{v^{\prime \prime}(t)}{v(t)} \bar{u}\right. \\
\\
\left.\quad-s^{2} f(\bar{u})-s^{2} g(s)\right] d s d \tau \mid .
\end{gathered}
$$

$$
I_{2} \leq \int_{\frac{1}{\alpha}}^{r_{1}}\left(I_{21}+I_{22}\right)^{p} d r
$$

For $I_{21}$ we have the following estimate ( here we use that $f(\bar{u}) \geq \frac{a}{2}|\bar{u}|^{2} \geq \frac{a}{4 A^{2}}|\bar{u}|$ for $r \in\left[\frac{1}{\alpha}, \frac{1}{\beta}\right]$ )

$$
\begin{gathered}
I_{21} \leq \int_{r}^{r+h} \frac{1}{\tau^{2}-K \tau+Q^{2}} \int_{\frac{1}{\alpha}}^{\frac{1}{\beta}}\left(\frac{s^{4}}{s^{2}-K s+Q^{2}} \frac{v^{\prime \prime}(t)}{v(t)} \bar{u}\right. \\
\left.-\frac{a}{4 A^{2}} s^{2} \bar{u}+C \frac{1}{A^{2}}\|\bar{u}\|_{\dot{B}_{p, q}^{\gamma}\left[0, r_{1}\right]}\right) d s d \tau= \\
=\int_{r}^{r+h} \frac{1}{\tau^{2}-K \tau+Q^{2}} \int_{\frac{1}{\alpha}}^{\frac{1}{\beta}}\left(\frac{s^{4}}{s^{2}-K s+Q^{2}} \frac{v^{\prime \prime}(t)}{v(t)} \frac{A^{2} \bar{u}}{A^{2}}\right. \\
\left.\quad-\frac{a}{4 A^{2}} s^{2} \frac{A^{2} \bar{u}}{A^{2}}+C \frac{1}{A^{2}}\|\bar{u}\|_{\dot{B}_{p, q}^{\gamma}\left[0, r_{1}\right]}\right) d s d \tau= \\
=\int_{r}^{r+h} \frac{1}{\tau^{2}-K \tau+Q^{2}} \int_{\frac{1}{\alpha}}^{\frac{1}{\beta}}\left(\left(\frac{s^{4}}{s^{2}-K s+Q^{2}} \frac{v^{\prime \prime}(t)}{v(t)} \frac{1}{A^{2}}\right.\right. \\
\left.\left.-s^{2} \frac{a}{4 A^{4}}\right) A^{2} \bar{u}+C \frac{1}{A^{2}}\|\bar{u}\|_{\dot{B}_{p, q}^{\gamma}\left[0, r_{1}\right]}\right) d s d \tau .
\end{gathered}
$$

From i4) we have that (for $s \in\left[\frac{1}{\alpha}, \frac{1}{\beta}\right]$ )

$$
\begin{gathered}
\frac{s^{4}}{s^{2}-K s+Q^{2}} \frac{v^{\prime \prime}(t)}{v(t)} \frac{1}{A^{2}}-\frac{a}{4 A^{2}} \frac{s^{2}}{A^{2}} \geq \\
\geq \frac{1}{\alpha^{2}} \frac{1}{1-\alpha K+\alpha^{2} Q^{2}} \frac{a}{4 A^{6}}-\frac{1}{\beta^{2}} \frac{a}{4 A^{4}}>0 .
\end{gathered}
$$

On the other hand we have $\bar{u} \geq \frac{1}{A^{2}}$ for every $t \in[0,1]$ and every $r \in\left[\frac{1}{\alpha}, \frac{1}{\beta}\right]$, therefore $A^{2} \bar{u} \geq 1$ and $A^{2} \bar{u} \leq A^{2 p} \bar{u}^{p}$. Consequently

$$
\begin{aligned}
I_{21} \leq & \int_{r}^{r+h} \frac{1}{\tau^{2}-K \tau+Q^{2}} \int_{\frac{1}{\alpha}}^{\frac{1}{\beta}}\left(\left(\frac{s^{4}}{s^{2}-K s+Q^{2}} \frac{v^{\prime \prime}(t)}{v(t)} \frac{1}{A^{2}}\right.\right. \\
& \left.\left.-a s^{2} \frac{1}{4 A^{4}}\right) A^{2 p} \bar{u}^{p}+C \frac{1}{A^{2}}\|\bar{u}\|_{\dot{B}_{p, q}^{\gamma}\left[0, r_{1}\right]}\right) d s d \tau .
\end{aligned}
$$

From i5) we have that

$$
\frac{1}{1-K+Q^{2}} \leq A \leq A^{2} .
$$


From here we get

$$
\begin{aligned}
I_{21} \leq & \int_{r}^{r+h} \frac{1}{1-K+Q^{2}} \int_{\frac{1}{\alpha}}^{\frac{1}{\beta}}\left[\left[\frac{v^{\prime \prime}(t)}{v(t)}-\frac{a}{4 A^{4}}\right] A^{2 p} \bar{u}^{p}+C \frac{1}{A^{2}}\|\bar{u}\|_{\dot{B}_{p, q}^{\gamma}\left[0, r_{1}\right]}\right] d s d \tau \leq \\
& \leq \frac{1}{1-K+Q^{2}}\left(\frac{v^{\prime \prime}(t)-\frac{a}{4 A^{4}} v}{v} A^{2 p} \int_{0}^{1} \bar{u}^{p} d s+C \frac{1}{A^{2}}\|\bar{u}\|_{\dot{B}_{p, q}^{\gamma}\left[0, r_{1}\right]}\right) h \leq \\
& \leq \frac{1}{1-K+Q^{2}}\left(\frac{v^{\prime \prime}(t)-\frac{a}{4 A^{4}} v}{v} A^{2 p}\|\bar{u}\|_{L^{p}}^{p}+C \frac{1}{A^{2}}\|\bar{u}\|_{\dot{B}_{p, q}^{\gamma}\left[0, r_{1}\right]}\right) h \leq \\
& \leq \frac{1}{\left(1-K+Q^{2}\right)^{2}}\left(\frac{v^{\prime \prime}(t)-\frac{a}{4 A^{4}} v}{v} A^{2 p}\|\bar{u}\|_{L^{p}}^{p}+C \frac{1}{A^{2}}\|\bar{u}\|_{\dot{B}_{p, q}^{\gamma}\left[0, r_{1}\right]}\right) h .
\end{aligned}
$$

After we use the lemma 1 we get

$$
I_{21} \leq \frac{1}{\left(1-K+Q^{2}\right)^{2}}\left(C^{p} \frac{v^{\prime \prime}(t)-\frac{a}{4 A^{4}} v}{v} A^{2 p}\|\bar{u}\|_{\dot{B}_{p, q}^{\gamma}}^{p}+C \frac{1}{A^{2}}\|\bar{u}\|_{\dot{B}_{p, q}^{\gamma}\left[0, r_{1}\right]}\right) h .
$$

For $I_{22}$ the following estimate

$$
I_{22} \leq\left(C \frac{1}{\left(1-K+Q^{2}\right)}\left(\frac{1}{1-K+Q^{2}} \frac{2 a}{A^{2}}+\frac{2 b}{A B}+\frac{1}{A^{2}}\right)\|\bar{u}\|_{\dot{B}_{p, q}^{\gamma}}\right) h .
$$

Consequently

$$
\begin{gathered}
I_{2} \leq\left[\left(\frac{C}{\left(1-K+Q^{2}\right)}\left(\frac{1}{1-K+Q^{2}} \frac{2 a}{A^{2}}+\frac{2 b}{A B}+\frac{1}{A^{2}}\right)\|\bar{u}\|_{\dot{B}_{p, q}^{\gamma}}\right) h+\right. \\
\left.+\frac{1}{\left(1-K+Q^{2}\right)^{2}}\left(C^{p} \frac{v^{\prime \prime}(t)-\frac{a}{4 A^{4}} v}{v} A^{2 p}\|\bar{u}\|_{\dot{B}_{p, q}^{\gamma}}^{p}+\frac{1}{A^{2}} C\|\bar{u}\|_{\dot{B}_{p, q}^{\gamma}\left[0, r_{1}\right]}\right) h\right]^{p},
\end{gathered}
$$

From (6) we get

$$
\begin{gathered}
\left\|\Delta_{h} \bar{u}\right\|_{L^{p}}^{q} \leq\left[\left[\left(C \frac{1}{\left(1-K+Q^{2}\right)}\left(\frac{1}{1-K+Q^{2}} \frac{2 a}{A^{2}}+\frac{2 b}{A B}+\frac{1}{A^{2}}\right)\|\bar{u}\|_{\dot{B}_{p, q}^{\gamma}}\right) h+\right.\right. \\
\left.+\frac{1}{\left(1-K+Q^{2}\right)^{2}}\left(C^{p} \frac{v^{\prime \prime}(t)-\frac{a}{4 A^{4}} v}{v} A^{2 p}\|\bar{u}\|_{\dot{B}_{p, q}^{\gamma}}^{p}+\frac{1}{A^{2}} C\|\bar{u}\|_{\dot{B}_{p, q}^{\gamma}\left[0, r_{1}\right]}\right) h\right]^{p}+ \\
\left.\left(C \frac{1}{1-K+Q^{2}}\left(\frac{1}{1-K+Q^{2}} \frac{2 a}{A^{2}}+\frac{2 b}{A B}+\frac{1}{A^{2}}\right)\|\bar{u}\|_{\dot{B}_{p, q}^{\gamma}} h\right)^{p}\right]^{\frac{q}{p}} .
\end{gathered}
$$

Then

$$
\begin{gathered}
\|\bar{u}\|_{\dot{B}_{p, q}^{\gamma}} \leq \frac{2^{2-\gamma}}{(q(1-\gamma))^{\frac{1}{q}}}\left(C \frac{1}{1-K+Q^{2}}\left(\frac{1}{1-K+Q^{2}} \frac{2 a}{A^{2}}+\frac{2 b}{A B}+\frac{1}{A^{2}}\right)\|\bar{u}\|_{\dot{B}_{p, q}^{\gamma}}\right)+ \\
+\frac{2^{1-\gamma}}{\left(1-K+Q^{2}\right)^{2}(q(1-\gamma))^{\frac{1}{q}}}\left(C^{p} \frac{v^{\prime \prime}(t)-\frac{a}{4 A^{4}} v}{v} A^{2 p}\|\bar{u}\|_{\dot{B}_{p, q}^{\gamma}}^{p}+\frac{1}{A^{2}} C\|\bar{u}\|_{\dot{B}_{p, q}^{\gamma}\left[0, r_{1}\right]}\right) .
\end{gathered}
$$

Let

$$
\begin{gathered}
C_{2}=C \frac{2^{2-\gamma}}{(q(1-\gamma))^{\frac{1}{q}}\left(1-K+Q^{2}\right)}\left(\frac{1}{1-K+Q^{2}} \frac{2 a}{A^{2}}+\frac{2 b}{A B}+\frac{1}{A^{2}}\right) \\
+C \frac{2^{1-\gamma}}{A^{2}\left(1-K+Q^{2}\right)^{2}(q(1-\gamma))^{\frac{1}{q}}}, \\
D_{1}=v\left(1-K+Q^{2}\right)^{2}(q(1-\gamma))^{\frac{1}{q}} \frac{1}{2^{1-\gamma} C^{p} A^{2 p}},
\end{gathered}
$$


From i3) we have that $C_{2}<1$. Then

$$
\|\bar{u}\|_{\dot{B}_{p, q}^{\gamma}} \leq C_{2}\|\bar{u}\|_{\dot{B}_{p, q}^{\gamma}}+\frac{v^{\prime \prime}(t)-\frac{a}{4 A^{4}} v}{D_{1}}\|\bar{u}\|_{\dot{B}_{p, q}^{\gamma}}^{p},
$$

from here

$$
\frac{\left(1-C_{2}\right) D_{1}}{v^{\prime \prime}(t)-\frac{a}{4 A^{4}} v} \leq\|\bar{u}\|_{\dot{B}_{p, q}^{\gamma}}^{p-1}
$$

Since

we have (from (7))

$$
\lim _{t \longrightarrow 0}\left[v^{\prime \prime}(t)-\frac{a}{4 A^{4}} v\right]=+0,
$$

$$
\lim _{t \longrightarrow 0}\|\bar{u}\|_{\dot{B}_{p, q}^{\gamma}}=\infty
$$

\section{Appendix}

Lemma 5.1. Let $\gamma \in(0,1), p>1, q \geq 1$. Then the set $N$ is closed subset of $\mathcal{C}\left((0,1] \dot{B}_{p, q}^{\gamma}\left(\mathcal{R}^{+}\right)\right)$.

Proof. Let $t \in(0,1]$ is fixed. Let $\left\{u_{n}\right\}$ is a sequence of elements of the set $N$ for which

$$
\lim _{n \rightarrow \infty}\left\|u_{n}-\tilde{\tilde{u}}\right\|_{\dot{B}_{p}^{\gamma}, q\left(\mathcal{R}^{+}\right)}=0,
$$

where $\tilde{\tilde{u}} \in \dot{B}_{p, q}^{\gamma}\left(\mathcal{R}^{+}\right)$. We have

$$
\lim _{n \longrightarrow \infty}\left\|u_{n}-\tilde{\tilde{u}}\right\|_{\dot{B}_{p, q}^{\gamma}\left(\left[0, r_{1}\right]\right)}=0 .
$$

We define $\tilde{u}$ as follows:

$$
\tilde{u}=\left\{\begin{array}{lll}
\tilde{\tilde{u}} & \text { for } & r \in\left[0, r_{1}\right] \\
0 & \text { for } & r>r_{1}
\end{array}\right.
$$

We have

$$
\lim _{n \longrightarrow \infty}\left\|u_{n}-\tilde{u}\right\|_{\dot{B}_{p, q}^{\gamma}\left(\left[0, r_{1}\right]\right)}=0 .
$$

First we note that for $u \in N R(u)$ is continuous function of $u$ and there exists $R^{\prime}(u)$ because $f(u) \in \mathcal{C}^{2}\left(\mathcal{R}^{1}\right)$. For $R^{\prime}(u)$ we have

$$
R^{\prime}(u)=\int_{r}^{r_{1}} \frac{1}{\tau^{2}-K \tau+Q^{2}} \int_{\tau}^{r_{1}}\left(\frac{s^{4}}{s^{2}-K s+Q^{2}} \frac{v^{\prime \prime}(t)}{v(t)}-s^{2} f^{\prime}(\xi) \bar{u}\right) d s d \tau .
$$

From here

$$
\begin{gathered}
\left|R^{\prime}(u)\right| \geq \int_{r}^{r_{1}} \frac{1}{\tau^{2}-K \tau+Q^{2}} \int_{\tau}^{r_{1}}\left(\frac{s^{4}}{s^{2}-K s+Q^{2}} \frac{v^{\prime \prime}(t)}{v(t)}-s^{2}\left|f^{\prime}(u)\right|\right) d s d \tau \geq \\
\geq \int_{r}^{r_{1}} \frac{1}{\tau^{2}-K \tau+Q^{2}} \int_{\tau}^{r_{1}}\left(\frac{s^{4}}{s^{2}-K s+Q^{2}} \frac{v^{\prime \prime}(t)}{v(t)}-a s^{2}|u|\right) d s d \tau \geq \\
\geq \int_{r}^{r_{1}} \frac{1}{\tau^{2}-K \tau+Q^{2}} \int_{\tau}^{r_{1}}\left(\frac{s^{4}}{s^{2}-K s+Q^{2}} \frac{v^{\prime \prime}(t)}{v(t)}-\frac{2 a}{A B} s^{2}\right) d s d \tau \geq \\
\geq \int_{r}^{r_{1}} \frac{1}{\tau^{2}-K \tau+Q^{2}} \int_{\tau}^{r_{1}}\left(\frac{s^{4}}{s^{2}-K s+Q^{2}} \frac{v^{\prime \prime}(t)}{v(t)}-\frac{2 a}{A B} r_{1}^{2}\right) d s d \tau \geq \\
\geq \int_{\frac{1}{\alpha}}^{\frac{1}{\beta}} \frac{1}{\tau^{2}-K \tau+Q^{2}} \int_{\tau}^{r_{1}} \frac{s^{4}}{s^{2}-K s+Q^{2}} \frac{v^{\prime \prime}(t)}{v(t)} d s d \tau-\frac{2 a}{\left(1-K+Q^{2}\right) A B} r_{1}^{4} \geq
\end{gathered}
$$


(here we use (H3))

$$
\begin{gathered}
\geq \int_{\frac{1}{\alpha}}^{\frac{1}{\beta}} \frac{1}{\tau^{2}-K \tau+Q^{2}} \int_{\tau}^{r_{1}} \frac{s^{4}}{s^{2}-K s+Q^{2}} \frac{a}{2 A^{4}} d s d \tau-\frac{2 a}{A B\left(1-K+Q^{2}\right)} r_{1}^{4} \geq \\
\geq\left(\frac{1}{\alpha}-\frac{1}{\beta}\right)^{2} \frac{1}{\left(1-\alpha K+\alpha^{2} Q^{2}\right)^{2}} \frac{a}{2 A^{4}}-\frac{2 a}{\left(1-K+Q^{2}\right) A B} r_{1}^{4}>0 .
\end{gathered}
$$

(in the last inequality we use the fifth inequality of i2)) From here for $u \in N$ exists

$$
M:=\min _{x \in\left[0, r_{1}\right]}\left|R^{\prime}(u)(x)\right|>0
$$

because $R^{\prime}(u)(x)$ is continuous function of $x \in\left[0, r_{1}\right]$.

Let

$$
M_{1}=\max _{r \in\left[0, r_{1}\right]}\left|\frac{\partial}{\partial r}\left(R^{\prime}(u)\right)(r)\right| .
$$

Now we will prove that

$$
\text { for } \begin{aligned}
\forall \epsilon>0 & \exists \delta=\delta(\epsilon)>0 \quad \text { : from }|x-y|<\delta \\
\Longrightarrow & \left|u_{m}(x)-u_{m}(y)\right|<\epsilon \quad \forall m .
\end{aligned}
$$

We suppose that there exists $\tilde{\epsilon}>0$ such that for every $\delta>0$ there exist natural $m$ and $x, y \in\left[0, r_{1}\right],|x-y|<\delta$ for which $\left|u_{m}(x)-u_{m}(y)\right| \geq \tilde{\epsilon}$. We choose $\tilde{\tilde{\epsilon}}>0$ such that $\tilde{\tilde{\epsilon}}<M \tilde{\epsilon}$. We note that $R\left(u_{m}\right)(x)$ is uniformly continuous function of $x \in\left[0, r_{1}\right]$ (For $u \in N$ the function $R(u)(r)$ is uniformly continuous function of $r \in\left[0, r_{1}\right]$ because $R(u)(r) \in \mathcal{C}^{2}\left(\left[0, r_{1}\right]\right)$ and as in point 2$)$ of the proof of theorem 2.1 we have $\left.\left|\frac{\partial}{\partial r} R(u)(r)\right| \leq \frac{2}{A B}\right)$. Then there exists $\delta_{1}=\delta_{1}(\tilde{\tilde{\epsilon}})>0$ such that for every $u \in N$

$$
|R(u)(x)-R(u)(y)|<\tilde{\tilde{\epsilon}} \quad \text { for } \quad \forall \quad x, y \in\left[0, r_{1}\right] \quad: \quad|x-y|<\delta_{1} .
$$

Then we may choose $0<\delta<\min \left\{\delta_{1}, \frac{(M \tilde{\epsilon}-\tilde{\tilde{\epsilon}}) A B}{2 M_{1}}\right\}$ such that there exist natural $m$ and $x_{1} \in\left[0, r_{1}\right], x_{2} \in\left[0, r_{1}\right]$ for which $\left|x_{1}-x_{2}\right|<\delta$ and $\left|u_{m}\left(x_{1}\right)-u_{m}\left(x_{2}\right)\right| \geq \tilde{\epsilon}$. In particular

$$
\left|R\left(u_{m}\right)\left(x_{1}\right)-R\left(u_{m}\right)\left(x_{2}\right)\right|<\tilde{\tilde{\epsilon}}
$$

Then from the midle point theorem we have

$$
\begin{aligned}
& R(0)=0, R\left(u_{m}\right)\left(x_{1}\right)=R^{\prime}(\xi)\left(x_{1}\right) u_{m}\left(x_{1}\right), \quad R\left(u_{m}\right)\left(x_{2}\right)=R^{\prime}(\xi)\left(x_{2}\right) u_{m}\left(x_{2}\right), \\
& \left|R\left(u_{m}\right)\left(x_{1}\right)-R\left(u_{m}\right)\left(x_{2}\right)\right|=\left|R^{\prime}(\xi)\left(x_{1}\right) u_{m}\left(x_{1}\right)-R^{\prime}(\xi)\left(x_{2}\right) u_{m}\left(x_{2}\right)\right|= \\
& =\left|R^{\prime}(\xi)\left(x_{1}\right) u_{m}\left(x_{1}\right)-R^{\prime}(\xi)\left(x_{1}\right) u_{m}\left(x_{2}\right)+R^{\prime}(\xi)\left(x_{1}\right) u_{m}\left(x_{2}\right)-R^{\prime}(\xi)\left(x_{2}\right) u_{m}\left(x_{2}\right)\right| \geq \\
& \geq\left|R^{\prime}(\xi)\left(x_{1}\right) u_{m}\left(x_{1}\right)-R^{\prime}(\xi)\left(x_{1}\right) u_{m}\left(x_{2}\right)\right|-\left|R^{\prime}(\xi)\left(x_{1}\right)-R^{\prime}(\xi)\left(x_{2}\right)\right|\left|u_{m}\left(x_{2}\right)\right|= \\
& =\left|R^{\prime}(\xi)\left(x_{1}\right)\right|\left|u_{m}\left(x_{1}\right)-u_{m}\left(x_{2}\right)\right|-\left|\frac{\partial}{\partial r}\left(R^{\prime}(\xi)\right)(\eta)\right|\left|x_{1}-x_{2}\right|\left|u_{m}\left(x_{2}\right)\right| \geq \\
& \geq M \tilde{\epsilon}-M_{1} \delta \frac{2}{A B} \geq \tilde{\tilde{\epsilon}},
\end{aligned}
$$

which is contradiction with (8).

Consequently

$$
\text { for } \begin{gathered}
\forall \epsilon>0 \quad \exists \delta=\delta(\epsilon)>0 \quad \text { : from }|x-y|<\delta \\
\Longrightarrow \quad\left|u_{m}(x)-u_{m}(y)\right|<\epsilon \quad \forall m .
\end{gathered}
$$

On the other hand, from the definition of the set $N$ we have

$$
\left|u_{m}\right| \leq \frac{2}{A B} \quad \forall m .
$$


From (9) and (10) we conclude that the set $\left\{u_{n}\right\}$ is compact subset of $\mathcal{C}\left(\left[0, r_{1}\right]\right)$. Then there exists subsequence $\left\{u_{n_{k}}\right\}$ and finction $u \in \mathcal{C}\left(\left[0, r_{1}\right]\right)$ for which: for every $\epsilon>0$ there exists $M=M(\epsilon)>0$ such that for every $n_{k}>M$ we have $\left|u_{n_{k}}(x)-u(x)\right|<\epsilon$ for every $x \in\left[0, r_{1}\right] ; u(x)=0$ for $x>r_{1}$. From here and from $\lim _{k \longrightarrow \infty}\left\|u_{n_{k}}-\tilde{u}\right\|_{\dot{B}_{p, q}^{\gamma}\left(\left[0, r_{1}\right]\right)}=0$ we have : for every $\epsilon>0 \exists M=M(\epsilon)>0$ such that for every $n_{k}>M$ we have

$$
\max _{x \in\left[0, r_{1}\right]}\left|u_{n_{k}}-u\right|<\epsilon, \quad\left\|u_{n_{k}}-\tilde{u}\right\|_{\dot{B}_{p, q}^{\gamma}\left(\left[0, r_{1}\right]\right)}<\epsilon .
$$

Then for every $n_{k}>M$ we have

$$
\begin{gathered}
|u-\tilde{u}| \leq\left|u-u_{n_{k}}\right|+\left|u_{n_{k}}-\tilde{u}\right|<\epsilon+\left|\tilde{u}-u_{n_{k}}\right|, \\
\int_{0}^{r_{1}}|u-\tilde{u}| d x<\epsilon r_{1}+\int_{0}^{r_{1}}\left|\tilde{u}-u_{n_{k}}\right| d x,
\end{gathered}
$$

(here we use the Hölder's inequality)

$$
\|u-\tilde{u}\|_{L^{1}\left[0, r_{1}\right]}<\epsilon r_{1}+r_{1}^{\frac{1}{q_{1}}}\left(\int_{0}^{r_{1}}\left|\tilde{u}-u_{n_{k}}\right|^{p} d x\right)^{\frac{1}{p}}, \frac{1}{p}+\frac{1}{q_{1}}=1,
$$

for $h>0$ we have

$$
\begin{gathered}
h^{-1-q \gamma}\|u-\tilde{u}\|_{L^{1}\left[0, r_{1}\right]}<h^{-1-q \gamma} \epsilon r_{1}+r_{1}^{\frac{1}{q_{1}}} h^{-1-q \gamma}\left(\int_{0}^{r_{1}}\left|\tilde{u}-u_{n_{k}}\right|^{p} d x\right)^{\frac{1}{p}}, \\
\int_{1}^{2} h^{-1-q \gamma} d h\|u-\tilde{u}\|_{L^{1}\left[0, r_{1}\right]}<\int_{1}^{2} h^{-1-q \gamma} d h \epsilon r_{1} \\
+r_{1}^{\frac{1}{q_{1}}} \int_{1}^{2} h^{-1-q \gamma}\left(\int_{0}^{r_{1}}\left|\tilde{u}-u_{n_{k}}\right|^{p} d x\right)^{\frac{1}{p}} d h, \\
\quad \frac{1-2^{-q \gamma}}{q \gamma}\|u-\tilde{u}\|_{L^{1}\left[0, r_{1}\right]}<\frac{1-2^{-q \gamma}}{q \gamma} \epsilon r_{1} \\
+r_{1}^{\frac{1}{q_{1}}} \int_{1}^{2} h^{-1-q \gamma}\left(\int_{0}^{r_{1}}\left|\tilde{u}-u_{n_{k}}\right|^{p} d x\right)^{\frac{1}{p}} d h \leq
\end{gathered}
$$

(here we use the Hölder's inequality)

$$
\leq \frac{1-2^{-q \gamma}}{q \gamma} \epsilon r_{1}+r_{1}^{\frac{1}{q_{1}}}\left(\int_{1}^{2} h^{(-1-q \gamma) q}\left(\int_{0}^{r_{1}}\left|\tilde{u}-u_{n_{k}}\right|^{p} d x\right)^{\frac{q}{p}} d h\right)^{\frac{1}{q}} \leq
$$

(since $h>1$ we have $h^{(-1-q \gamma) q} \leq h^{-1-q \gamma}$ )

$$
\leq \frac{1-2^{-q \gamma}}{q \gamma} \epsilon r_{1}+r_{1}^{\frac{1}{q_{1}}}\left(\int_{1}^{2} h^{-1-q \gamma}\left(\int_{0}^{r_{1}}\left|\tilde{u}-u_{n_{k}}\right|^{p} d x\right)^{\frac{q}{p}} d h\right)^{\frac{1}{q}}=
$$

(here we use that for $x>r_{1}$ we have $u_{n_{k}}(x)=\tilde{u}(x)=0$ )

$$
\begin{gathered}
=\frac{1-2^{-q \gamma}}{q \gamma} \epsilon r_{1}+r_{1}^{\frac{1}{q_{1}}}\left(\int_{1}^{2} h^{-1-q \gamma}\left(\int_{0}^{r_{1}}\left|\Delta_{h}\left(\tilde{u}-u_{n_{k}}\right)\right|^{p} d x\right)^{\frac{q}{p}} d h\right)^{\frac{1}{q}} \leq \\
\leq \frac{1-2^{-q \gamma}}{q \gamma} \epsilon r_{1}+r_{1}^{\frac{1}{q_{1}}}\left(\int_{0}^{2} h^{-1-q \gamma}\left(\int_{0}^{r_{1}}\left|\Delta_{h}\left(\tilde{u}-u_{n_{k}}\right)\right|^{p} d x\right)^{\frac{q}{p}} d h\right)^{\frac{1}{q}}= \\
=\frac{1-2^{-q \gamma}}{q \gamma} \epsilon r_{1}+r_{1}^{\frac{1}{q_{1}}}|| \tilde{u}-u_{n_{k}} \|_{\dot{B}_{p, q}^{\gamma}\left(\left[0, r_{1}\right]\right)}< \\
<\epsilon\left(\frac{1-2^{-q \gamma}}{q \gamma} r_{1}+r_{1}^{\frac{1}{q_{1}}}\right),
\end{gathered}
$$


i.e.

$$
\frac{1-2^{-q \gamma}}{q \gamma}\|u-\tilde{u}\|_{L^{1}\left[0, r_{1}\right]}<\epsilon\left(\frac{1-2^{-q \gamma}}{q \gamma} r_{1}+r_{1}^{\frac{1}{q_{1}}}\right)
$$

for every $\epsilon>0$. Consequently $u=\tilde{u}$ a.e.(almost everywhere) in $\left[0, r_{1}\right],|u|^{p}=|\tilde{u}|^{p}$ a.e. in $\left[0, r_{1}\right]$. From here $\left|u_{n}-u\right|=\left|u_{n}-\tilde{u}\right|$ a.e., $\left|u_{n}-u\right|^{p}=\left|u_{n}-\tilde{u}\right|^{p}$ a.e. in $\left[0, r_{1}\right]$. Since $u_{n}(x)=u(x)=0$ for $x>r_{1}$ we have $\left|\Delta_{h}\left(u_{n}-u\right)\right|^{p}=\left|\Delta_{h}\left(u_{n}-\tilde{u}\right)\right|^{p}$, $\left|\Delta_{h} u\right|^{p}=\left|\Delta_{h} \tilde{u}\right|^{p}$ a.e. in $\left[0, r_{1}\right]$, for $h>0$. Therefore $u \in \dot{B}_{p, q}^{\gamma}\left(\left[0, r_{1}\right]\right)$ and

$$
\int_{0}^{r_{1}}\left|u_{n}-u\right|^{p} d x=\int_{0}^{r_{1}}\left|u_{n}-\tilde{u}\right|^{p} d x
$$

Now we will see that $\lim _{n \longrightarrow \infty}\left\|u_{n}-u\right\|_{\dot{B}_{p, q}^{\gamma}\left(\left[0, r_{1}\right]\right)}=0$. Really,

$$
\begin{gathered}
\left\|u_{n}-u\right\|_{\dot{B}_{p, q}^{\gamma}\left(\left[0, r_{1}\right]\right)}=\left(\int_{0}^{2} h^{-1-q \gamma}\left(\int_{0}^{r_{1}}\left|\Delta_{h}\left(u_{n}-u\right)\right|^{p} d x\right)^{\frac{q}{p}} d h\right)^{\frac{1}{q}}= \\
=\left(\int_{0}^{2} h^{-1-q \gamma} \int_{0}^{r_{1}}\left|\Delta_{h}\left(u_{n}-\tilde{u}\right)\right|^{q} d x d h\right)^{\frac{1}{q}}= \\
\left\|u_{n}-\tilde{u}\right\|_{\dot{B}_{p, q}^{\gamma}\left(\left[0, r_{1}\right]\right)} \longrightarrow n \longrightarrow \infty 0 .
\end{gathered}
$$

Consequently for every sequence $\left\{u_{n}\right\}$ with elements from $N$, which converges in $\dot{B}_{p, q}^{\gamma}\left(\left[0, r_{1}\right]\right)$ there exists function $u \in \mathcal{C}\left(\left[0, r_{1}\right]\right), u \in \dot{B}_{p, q}^{\gamma}\left(\left[0, r_{1}\right]\right)$, for which $\| u_{n}-$ $u \|_{\dot{B}_{p, q}^{\gamma}\left(\left[0, r_{1}\right]\right)} \longrightarrow n \longrightarrow \infty 0$.

Below we suppose that the sequence $\left\{u_{n}\right\}$ is a sequence of elements of the set $N$ which converges in $\dot{B}_{p, q}^{\gamma}\left(\left[0, r_{1}\right]\right)$. Then there exists $u \in \mathcal{C}\left(\left[0, r_{1}\right]\right), u(x)=0$ for $x>r_{1}, u \in \dot{B}_{p, q}^{\gamma}\left(\left[0, r_{1}\right]\right),\left\|u_{n}-u\right\|_{\dot{B}_{p, q}^{\gamma}\left(\left[0, r_{1}\right]\right)} \longrightarrow_{n \longrightarrow \infty} 0$.

Now we suppose that $u\left(r_{1}\right) \neq 0$. Since $u \in \mathcal{C}\left(\left[0, r_{1}\right]\right), u_{n} \in \mathcal{C}\left(\left[0, r_{1}\right]\right), u_{n}\left(r_{1}\right)=0$ for every natural $n$, there exist $\epsilon_{2}>0$ and $\Delta_{1} \subset\left[0, r_{1}\right], r_{1} \in \Delta_{1}$, such that

$$
\left|u_{n}\right|<\frac{\epsilon_{2}}{2},|u|>\epsilon_{2}
$$

for every natural $n$ and every $x \in \Delta_{1}$. Then for every natural $n$ and for every $x \in \Delta_{1}$ we have

Let $\epsilon_{3}>0$ is such that

$$
\left|u_{n}(x)-u(x)\right|>\frac{\epsilon_{2}}{2} .
$$

$$
\epsilon_{3}<\frac{\epsilon_{2}}{2} \frac{1-2^{-q \gamma}}{q \gamma} \mu\left(\Delta_{1}\right) r_{1}^{-\frac{1}{q_{1}}}, \quad \frac{1}{p}+\frac{1}{q_{1}}=1,
$$

where $\mu\left(\Delta_{1}\right)$ is the measure of the set $\Delta_{1}$. There exists $M>0$ such that for every $n>M$ we have $\left\|u_{n}-u\right\|_{\dot{B}_{p, q}^{\gamma}\left(\left[0, r_{1}\right]\right)}<\epsilon_{3}$. Consequently for every $n>M$ and for every $x \in \Delta_{1}$ we have

$$
\left|u_{n}(x)-u(x)\right|>\frac{\epsilon_{2}}{2}, \quad\left\|u_{n}-u\right\|_{\dot{B}_{p, q}^{\gamma}\left(\left[0, r_{1}\right]\right)}<\epsilon_{3} .
$$

Also we have

$$
\frac{\epsilon_{2}}{2} \mu\left(\Delta_{1}\right)<\int_{\Delta_{1}}\left|u_{n}(x)-u(x)\right| d x \leq \int_{0}^{r_{1}}\left|u_{n}(x)-u(x)\right| d x \leq
$$

(here we use the Hölder's inequality)

$$
\leq\left(\int_{0}^{r_{1}}\left|u_{n}(x)-u(x)\right|^{p} d x\right)^{\frac{1}{p}} r_{1}^{\frac{1}{q_{1}}} .
$$


For $h>0$ we have

$$
\begin{gathered}
h^{-1-q \gamma} \frac{\epsilon_{2}}{2} \mu\left(\Delta_{1}\right) \leq h^{-1-q \gamma}\left(\int_{0}^{r_{1}}\left|u_{n}(x)-u(x)\right|^{p} d x\right)^{\frac{1}{p}} r_{1}^{\frac{1}{q_{1}}} \\
\int_{1}^{2} h^{-1-q \gamma} \frac{\epsilon_{2}}{2} \mu\left(\Delta_{1}\right) d h \leq \int_{1}^{2} h^{-1-q \gamma}\left(\int_{0}^{r_{1}}\left|u_{n}(x)-u(x)\right|^{p} d x\right)^{\frac{1}{p}} r_{1}^{\frac{1}{q_{1}}} d h,
\end{gathered}
$$

(here we use the Hölder's inequality)

$$
\frac{1-2^{-q \gamma}}{q \gamma} \frac{\epsilon_{2}}{2} \mu\left(\Delta_{1}\right) \leq\left(\int_{1}^{2} h^{(-1-q \gamma) q}\left(\int_{0}^{r_{1}}\left|u_{n}(x)-u(x)\right|^{p} d x\right)^{\frac{q}{p}} d h\right)^{\frac{1}{q}} r_{1}^{\frac{1}{q_{1}}} \leq
$$

(since $h>1$ we have $h^{(-1-q \gamma) q} \leq h^{-1-q \gamma}$ )

$$
\leq\left(\int_{1}^{2} h^{-1-q \gamma}\left(\int_{0}^{r_{1}}\left|u_{n}(x)-u(x)\right|^{q} d x\right)^{\frac{q}{p}} d h\right)^{\frac{1}{q}} r_{1}^{\frac{1}{q_{1}}} \leq
$$

(here we use that $u_{n}=u=0$ for $x>r_{1}$ )

$$
\begin{gathered}
\leq\left(\int_{1}^{2} h^{-1-q \gamma}\left(\int_{0}^{r_{1}}\left|\Delta_{h}\left(u_{n}(x)-u(x)\right)\right|^{p} d x\right)^{\frac{q}{p}} d h\right)^{\frac{1}{q}} r_{1}^{\frac{1}{q_{1}}} \leq \\
\leq\left(\int_{0}^{2} h^{-1-q \gamma}\left(\int_{0}^{r_{1}}\left|\Delta_{h}\left(u_{n}(x)-u(x)\right)\right|^{p} d x\right)^{\frac{q}{p}} d h\right)^{\frac{1}{q}} r_{1}^{\frac{1}{q_{1}}}= \\
\left\|u_{n}-u\right\|_{\dot{B}_{p, q}^{\gamma}\left(\left[0, r_{1}\right]\right)} r_{1}^{\frac{1}{q_{1}}}< \\
<\epsilon_{3} r_{1}^{\frac{1}{q_{1}}} .
\end{gathered}
$$

Therefore

$$
\frac{1-2^{-q \gamma}}{q \gamma} \frac{\epsilon_{2}}{2} \mu\left(\Delta_{1}\right)<\epsilon_{3} r_{1}^{\frac{1}{q_{1}}}
$$

which is a contradiction with (11). Consequently $u\left(r_{1}\right)=0$. From here $u(t, r)=0$ for $r \geq r_{1}$. Then $u_{r}(t, r)=u_{r r}(t, r)=0$ for every $r \geq r_{1}$.

Now we suppose that the

$$
|u(t, r)| \leq \frac{2}{A B}
$$

is not hold for every $r \in\left[0, r_{1}\right]$. Since $u \in \mathcal{C}\left(\left[0, r_{1}\right]\right)$ we may take $\epsilon_{4}>0$ and $\Delta_{2} \subset\left[0, r_{1}\right]$ such that

$$
|u| \geq \frac{2}{A B}+\epsilon_{4} \quad \text { for } \quad r \in \Delta_{2} .
$$

Then for every natural $n$ and for every $r \in \Delta_{2}$ we have

$$
\left|u_{n}-u\right| \geq|u|-\left|u_{n}\right| \geq \frac{2}{A B}+\epsilon_{4}-\frac{2}{A B}=\epsilon_{4} .
$$

Let $\epsilon_{5}>0$ is such that

$$
\frac{1-2^{-q \gamma}}{q \gamma} \epsilon_{4} \mu\left(\Delta_{2}\right)>\epsilon_{5} r_{1}^{\frac{1}{q_{1}}} .
$$

There exist $M>0$ such that for every $n>M$ we have $\left\|u_{n}-u\right\|_{\left.\dot{B}_{p}^{\gamma}, q\left(0, r_{1}\right]\right)}<\epsilon_{5}$. Consequently for every $n>M$ and for every $x \in \Delta_{2}$ we have

$$
\left|u_{n}(x)-u(x)\right| \geq \epsilon_{4}, \quad\left\|u_{n}-u\right\|_{\dot{B}_{p, q}^{\gamma}\left(\left[0, r_{1}\right]\right)}<\epsilon_{5} .
$$


Also we have

$$
\epsilon_{4} \mu\left(\Delta_{2}\right)<\int_{\Delta_{2}}\left|u_{n}(x)-u(x)\right| d x \leq
$$

(here we use the Hölder's inequality)

$$
\leq\left(\int_{0}^{r_{1}}\left|u_{n}(x)-u(x)\right|^{p} d x\right)^{\frac{1}{p}} r_{1}^{\frac{1}{q_{1}}}
$$

For $h>0$ we have

$$
\begin{gathered}
h^{-1-q \gamma} \epsilon_{4} \mu\left(\Delta_{2}\right) \leq h^{-1-q \gamma}\left(\int_{0}^{r_{1}}\left|u_{n}(x)-u(x)\right|^{p} d x\right)^{\frac{1}{p}} r_{1}^{\frac{1}{q_{1}}}, \\
\int_{1}^{2} h^{-1-q \gamma} \epsilon_{4} \mu\left(\Delta_{2}\right) d h \leq \int_{1}^{2} h^{-1-q \gamma}\left(\int_{0}^{r_{1}}\left|u_{n}(x)-u(x)\right|^{p} d x\right)^{\frac{1}{p}} r_{1}^{\frac{1}{q_{1}}} d h,
\end{gathered}
$$

(here we use the Hölder's inequality)

$$
\frac{1-2^{-q \gamma}}{q \gamma} \epsilon_{4} \mu\left(\Delta_{2}\right) \leq\left(\int_{1}^{2} h^{(-1-q \gamma) q}\left(\int_{0}^{r_{1}}\left|u_{n}(x)-u(x)\right|^{p} d x\right)^{\frac{q}{p}} d h\right)^{\frac{1}{q}} r_{1}^{\frac{1}{q_{1}}} \leq
$$

(since $h>1$ we have $h^{(-1-q \gamma) q} \leq h^{-1-q \gamma}$ )

$$
\leq\left(\int_{1}^{2} h^{-1-q \gamma}\left(\int_{0}^{r_{1}}\left|u_{n}(x)-u(x)\right|^{p} d x\right)^{\frac{q}{p}} d h\right)^{\frac{1}{q}} r_{1}^{\frac{1}{q_{1}}} \leq
$$

(here we use that $u_{n}=u=0$ for $x>r_{1}$ )

$$
\begin{gathered}
\leq\left(\int_{1}^{2} h^{-1-q \gamma}\left(\int_{0}^{r_{1}}\left|\Delta_{h}\left(u_{n}(x)-u(x)\right)\right|^{p} d x\right)^{\frac{q}{p}} d h\right)^{\frac{1}{q}} r_{1}^{\frac{1}{q_{1}}} \leq \\
\leq\left(\int_{0}^{2} h^{-1-q \gamma}\left(\int_{0}^{r_{1}}\left|\Delta_{h}\left(u_{n}(x)-u(x)\right)\right|^{p} d x\right)^{\frac{q}{p}} d h\right)^{\frac{1}{q}} r_{1}^{\frac{1}{q_{1}}}= \\
\left\|u_{n}-u\right\|_{\dot{B}_{p, q}^{\gamma}\left(\left[0, r_{1}\right]\right)} r_{1}^{\frac{1}{q_{1}}}< \\
<\epsilon_{5} r_{1}^{\frac{1}{q_{1}}} .
\end{gathered}
$$

Therefore

$$
\frac{1-2^{-q \gamma}}{q \gamma} \epsilon_{4} \mu\left(\Delta_{2}\right)<\epsilon_{5} r_{1}^{\frac{1}{q_{1}}},
$$

which is a contradiction with (12). Therefore $|u| \leq \frac{2}{A B}$ for every $r \in\left[0, r_{1}\right]$.

Now we suppose that the inequality

$$
|u(t, r)| \geq \frac{1}{A^{2}}
$$

is not true for every $r \in\left[\frac{1}{\alpha}, \frac{1}{\beta}\right]$. Since $u \in \mathcal{C}\left(\left[0, r_{1}\right]\right)$ we may take $\epsilon_{6}>0$ and $\Delta_{3} \subset\left[\frac{1}{\alpha}, \frac{1}{\beta}\right]$ such that

$$
|u| \leq \frac{1}{A^{2}}-\epsilon_{6} \quad \text { for } \quad r \in \Delta_{3} .
$$

Then for every natural $n$ and for every $r \in \Delta_{3}$ we have

$$
\left|u_{n}-u\right| \geq\left|u_{n}\right|-|u| \geq \frac{1}{A^{2}}+\epsilon_{6}-\frac{1}{A^{2}}=\epsilon_{6} .
$$


Let $\epsilon_{7}>0$ is such that

$$
\frac{1-2^{-q \gamma}}{q \gamma} \epsilon_{6} \mu\left(\Delta_{3}\right)>\epsilon_{7} r_{1}^{\frac{1}{q_{1}}} .
$$

There exist $M>0$ such that for every $n>M$ we have $\left\|u_{n}-u\right\|_{\dot{B}_{p, q}^{\gamma}\left(\left[0, r_{1}\right]\right)}<\epsilon_{7}$. Consequently for every $n>M$ and for every $x \in \Delta_{3}$ we have

$$
\left|u_{n}(x)-u(x)\right|>\epsilon_{6}, \quad\left\|u_{n}-u\right\|_{\dot{B}_{p, q}^{\gamma}\left(\left[0, r_{1}\right]\right)}<\epsilon_{7} .
$$

Also we have

$$
\epsilon_{6} \mu\left(\Delta_{3}\right)<\int_{\Delta_{3}}\left|u_{n}(x)-u(x)\right| d x \leq
$$

(here we use the Hölder's inequality)

$$
\leq\left(\int_{0}^{r_{1}}\left|u_{n}(x)-u(x)\right|^{p} d x\right)^{\frac{1}{p}} r_{1}^{\frac{1}{q_{1}}} .
$$

For $h>0$ we have

$$
\begin{gathered}
h^{-1-q \gamma} \epsilon_{6} \mu\left(\Delta_{3}\right) \leq h^{-1-q \gamma}\left(\int_{0}^{r_{1}}\left|u_{n}(x)-u(x)\right|^{p} d x\right)^{\frac{1}{p}} r_{1}^{\frac{1}{q_{1}}}, \\
\int_{1}^{2} h^{-1-q \gamma} \epsilon_{6} \mu\left(\Delta_{3}\right) d h \leq \int_{1}^{2} h^{-1-q \gamma}\left(\int_{0}^{r_{1}}\left|u_{n}(x)-u(x)\right|^{p} d x\right)^{\frac{1}{p}} r_{1}^{\frac{1}{q_{1}}} d h,
\end{gathered}
$$

(here we use the Hölder's inequality)

$$
\frac{1-2^{-q \gamma}}{q \gamma} \epsilon_{6} \mu\left(\Delta_{3}\right) \leq\left(\int_{1}^{2} h^{(-1-q \gamma) q}\left(\int_{0}^{r_{1}}\left|u_{n}(x)-u(x)\right|^{p} d x\right)^{\frac{q}{p}} d h\right)^{\frac{1}{q}} r_{1}^{\frac{1}{q_{1}}} \leq
$$

(since $h>1$ we have $h^{(-1-q \gamma) q} \leq h^{-1-q \gamma}$ )

$$
\leq\left(\int_{1}^{2} h^{-1-q \gamma}\left(\int_{0}^{r_{1}}\left|u_{n}(x)-u(x)\right|^{p} d x\right)^{\frac{q}{p}} d h\right)^{\frac{1}{q}} r_{1}^{\frac{1}{q_{1}}} \leq
$$

(here we use that $u_{n}=u=0$ for $x>r_{1}$ )

$$
\begin{gathered}
\leq\left(\int_{1}^{2} h^{-1-q \gamma}\left(\int_{0}^{r_{1}}\left|\Delta_{h}\left(u_{n}(x)-u(x)\right)\right|^{p} d x\right)^{\frac{q}{p}} d h\right)^{\frac{1}{q}} r_{1}^{\frac{1}{q_{1}}} \leq \\
\leq\left(\int_{0}^{2} h^{-1-q \gamma}\left(\int_{0}^{r_{1}}\left|\Delta_{h}\left(u_{n}(x)-u(x)\right)\right|^{p} d x\right)^{\frac{q}{p}} d h\right)^{\frac{1}{q}} r_{1}^{\frac{1}{q_{1}}}= \\
\left\|u_{n}-u\right\|_{\dot{B}_{p, q}^{\gamma}\left(\left[0, r_{1}\right]\right)_{1}}{ }^{\frac{1}{q_{1}}}< \\
<\epsilon_{7} r_{1}^{\frac{1}{q_{1}}} .
\end{gathered}
$$

Therefore

$$
\frac{1-2^{-q \gamma}}{q \gamma} \epsilon_{6} \mu\left(\Delta_{2}\right)<\epsilon_{7} r_{1}^{\frac{1}{q_{1}}},
$$

which is a contradiction with (13). Therefore $|u| \geq \frac{1}{A^{2}}$ for every $r \in\left[\frac{1}{\alpha}, \frac{1}{\beta}\right]$.

Now we suppose that the inequality

$$
u(t, r) \geq 0
$$


is not true for every $r \in\left[\frac{1}{\alpha}, r_{1}\right]$. Then from $u \in \mathcal{C}\left(\left[0, r_{1}\right]\right)$ and from $u_{n} \geq 0$ for every natural $n$ and for every $r \in\left[\frac{1}{\alpha}, r_{1}\right]$, we may take $\epsilon_{8}>0$ and $\Delta_{4} \subset\left[\frac{1}{\alpha}, r_{1}\right]$ such that for every natural $n$ and for every $r \in \Delta_{4}$ we have

$$
\left|u_{n}-u\right| \geq \epsilon_{8} .
$$

Let $\epsilon_{9}>0$ is such that

$$
\frac{1-2^{-q \gamma}}{q \gamma} \epsilon_{8} \mu\left(\Delta_{3}\right)>\epsilon_{9} r_{1}^{\frac{1}{q_{1}}}
$$

There exist $M>0$ such that for every $n>M$ we have $\left\|u_{n}-u\right\|_{\dot{B}_{p, q}^{\gamma}\left(\left[0, r_{1}\right]\right)}<\epsilon_{9}$. Consequently for every $n>M$ and for every $x \in \Delta_{4}$ we have

$$
\left|u_{n}(x)-u(x)\right|>\epsilon_{8}, \quad\left\|u_{n}-u\right\|_{\dot{B}_{p, q}^{\gamma}\left(\left[0, r_{1}\right]\right)}<\epsilon_{9} .
$$

Also we have

$$
\epsilon_{8} \mu\left(\Delta_{4}\right)<\int_{\Delta_{4}}\left|u_{n}(x)-u(x)\right| d x \leq
$$

(here we use the Hölder's inequality)

$$
\leq\left(\int_{0}^{r_{1}}\left|u_{n}(x)-u(x)\right|^{p} d x\right)^{\frac{1}{p}} r_{1}^{\frac{1}{q_{1}}}
$$

For $h>0$ we have

$$
\begin{gathered}
h^{-1-q \gamma} \epsilon_{8} \mu\left(\Delta_{4}\right) \leq h^{-1-q \gamma}\left(\int_{0}^{r_{1}}\left|u_{n}(x)-u(x)\right|^{p} d x\right)^{\frac{1}{p}} r_{1}^{\frac{1}{q_{1}}}, \\
\int_{1}^{2} h^{-1-q \gamma} \epsilon_{8} \mu\left(\Delta_{4}\right) d h \leq \int_{1}^{2} h^{-1-q \gamma}\left(\int_{0}^{r_{1}}\left|u_{n}(x)-u(x)\right|^{p} d x\right)^{\frac{1}{p}} r_{1}^{\frac{1}{q_{1}}} d h,
\end{gathered}
$$

(here we use the Hölder's inequality)

$$
\frac{1-2^{-q \gamma}}{q \gamma} \epsilon_{8} \mu\left(\Delta_{4}\right) \leq\left(\int_{1}^{2} h^{(-1-q \gamma) q}\left(\int_{0}^{r_{1}}\left|u_{n}(x)-u(x)\right|^{p} d x\right)^{\frac{q}{p}} d h\right)^{\frac{1}{q}} r_{1}^{\frac{1}{q_{1}}} \leq
$$

(since $h>1$ we have $h^{(-1-q \gamma) q} \leq h^{-1-q \gamma}$ )

$$
\leq\left(\int_{1}^{2} h^{-1-q \gamma}\left(\int_{0}^{r_{1}}\left|u_{n}(x)-u(x)\right|^{p} d x\right)^{\frac{q}{p}} d h\right)^{\frac{1}{q}} r_{1}^{\frac{1}{q_{1}}} \leq
$$

(here we use that $u_{n}=u=0$ for $x>r_{1}$ )

$$
\begin{gathered}
\leq\left(\int_{1}^{2} h^{-1-q \gamma}\left(\int_{0}^{r_{1}}\left|\Delta_{h}\left(u_{n}(x)-u(x)\right)\right|^{p} d x\right)^{\frac{q}{p}} d h\right)^{\frac{1}{q}} r_{1}^{\frac{1}{q_{1}}} \leq \\
\leq\left(\int_{0}^{2} h^{-1-q \gamma}\left(\int_{0}^{r_{1}}\left|\Delta_{h}\left(u_{n}(x)-u(x)\right)\right|^{p} d x\right)^{\frac{q}{p}} d h\right)^{\frac{1}{q}} r_{1}^{\frac{1}{q_{1}}}= \\
\left\|u_{n}-u\right\|_{\dot{B}_{p, q}^{\gamma}\left(\left[0, r_{1}\right]\right]} r_{1}^{\frac{1}{q_{1}}}< \\
<\epsilon_{9} r_{1}^{\frac{1}{q_{1}}} .
\end{gathered}
$$

Therefore

$$
\frac{1-2^{-q \gamma}}{q \gamma} \epsilon_{8} \mu\left(\Delta_{4}\right)<\epsilon_{9} r_{1}^{\frac{1}{q_{1}}},
$$

which is a contradiction with (14). Therefore $|u| \geq 0$ for every $r \in\left[\frac{1}{\alpha}, r_{1}\right]$.

Consequently $u \in N$. 
Then for every sequence $\left\{u_{n}\right\} \subset N$, which converges in $\dot{B}_{p, q}^{\gamma}\left(\left[0, r_{1}\right]\right)$ there exists $u \in N$ for which

$$
\lim _{n \longrightarrow \infty}\left\|u_{n}-u\right\|_{\dot{B}_{p, q}^{\gamma}\left(\left[0, r_{1}\right]\right)}=0 . \bullet
$$

As in the proof of lemma 4.1 it is easy to verify that the set $N_{1}$ is closed subset of the space $\mathcal{C}\left((0,1] \dot{B}_{p, q}^{\gamma}\left(\mathcal{R}^{+}\right)\right)$for $\gamma \in(0,1), p>1, q \geq 1$.

\section{References}

[1] Shatah, J., M. Struwe. Geometric wave equation. Courant lecture notes in mathematics 2(1998).

[2] Taylor, M. Partial differential equations III. Springer-Verlag, 1996.

[3] Runst, T., W. Sickel. Sobolev spaces of fractional order, Nemytskij operators and nonlinear partial differential equations, 1996, New York.

[4] Georgiev, S. Blow up of solutions for Klein-Gordon equations in the Reissner-Nordström metric, EJDE, Vol 2005(2005), No 67, pp. 1-22.

[5] Rauch, J. The $u^{5}-$ Klein - Gordon equation, Brezis. and Lions. eds., Pilman Research Notes in Math. 53, pp. 335-364.

[6] Jörgens, K. Das Anfangswertproblem im Grossen für eine Klasse nicht-linear Wellengleichungen, Math. Z., 77, 1961, pp. 295-308.

[7] John, F. Blow up of solutions of nonlinear wave equations in three space dimensions, Manuscripta Math. 28, 1979, 235-268.

University of Sofia, Faculty of Mathematics and Informatics, Department of DifFerential Equations, Bulgaria

E-mail address: sgg2000bg@yahoo.com 